\title{
A review of statistical models for the break-up of an immiscible fluid immersed into a fully developed turbulent flow
}

\author{
J.C. Lasheras ，C. Eastwood , C. Martínez-Bazán ，J.L. Montañés \\ Department of Mechanical and Aerospace Engineering, University of California at San Diego, 9500 Gilman Drive, \\ La Jolla, CA 92093-0411, USA \\ Departamento de Ingenieria Mecánica, Universidad Carlos II de Madrid, ClButarque 15, 28911 Leganés, \\ Madrid, Spain \\ Departamento de Motopropulsión y Termofluidodinámica, ETSI Aeronáuticos, Pz Cardenal Cisneros 3 , \\ 28040 Madrid, Spain
}

\begin{abstract}
We consider the statistical description of the break-up of an immiscible fluid lump immersed into a fully developed turbulent flow. We focus on systems where there is no relative velocity between the continuous and dispersed phases. In this case, particle fragmentation is caused only by turbulent velocity fluctuations. The most relevant models proposed for the particle break-up frequency and for the shape of the daughter particle size distribution are reviewed. Their predictions are compared to recent experimental data, obtained for the break-up of an air cavity immersed into a high Reynolds number, turbulent water jet. Models based on purcly kinematic arguments show the best agreement with the experimental data.
\end{abstract}

Keywords: Turbulence; Two-phasc flows; Atomization; Brcak-up; Drops; Bubbles

\section{Introduction}

A statistical description of a dispersed, two-phase flow can be obtained by means of a distribution function $p(D, \mathbf{x}, \mathbf{v}, t) \mathrm{d} D \mathrm{~d} \mathbf{x} \mathrm{d} \mathbf{v}$, defined as the probable number of particles with diameters in the range $\mathrm{d} D$ about $D$, located in the spatial range $\mathrm{d} \mathbf{x}$ about the position $\mathbf{x}$, with a velocity range $\mathrm{d} \mathbf{v}$ about $\mathbf{v}$, at time $t$. A Boltzmann-type equation, often referred to as the population balance 
equation, can be written to describe the temporal and spatial rate of change of the distribution function $p$

$$
\frac{\partial p}{\partial t}+\nabla_{\mathbf{x}} \cdot(\mathbf{v} p)+\nabla_{\mathbf{v}} \cdot(\mathbf{F} p)=-\frac{\partial}{\partial D}(R p)+\dot{Q}_{\mathrm{b}}^{\prime}+\dot{Q}_{c}^{\prime}+\Gamma,
$$

where the rates of change of $p$ with time due to break-up and coalescence are denoted by $\dot{Q}_{\mathrm{b}}^{\prime}$ and $\dot{Q}_{c}$, respectively. The force per unit mass acting on a particle is denoted by $\mathbf{F}$, and the rate of change with time of its diameter due to evaporation, condensation, or dissolution is given by $R . \Gamma$ represents the rate of change of the distribution function caused by collisions which do not result in coalescence. Integrating over the whole velocity space to eliminate the velocity dependence, one obtains the following (Williams, 1985):

$$
\frac{\partial n}{\partial t}+\nabla_{\mathbf{x}} \cdot(\overline{\mathbf{v}} n)=-\frac{\partial}{\partial D}(R n)+\int \dot{Q}_{\mathrm{b}}^{\prime} \mathrm{d} \mathbf{v}+\int \dot{Q}_{\mathrm{c}}^{\prime} \mathrm{d} \mathbf{v},
$$

where $n(D, \mathbf{x}, t)=\int p \mathrm{~d} \mathbf{v} . n(D, \mathbf{x}, t) \mathrm{d} D \mathrm{~d} \mathbf{x}$ denotes the probable number of particles with size in the range $\mathrm{d} D$ about $D$, in the spatial range $\mathrm{d} \mathbf{x}$ about $\mathbf{x}$ at time $t, \overline{\mathbf{v}}$ is the mean velocity of all particles of size $D$ at a location $\mathbf{x}$ at time $t$. Eq. (2) is usually expressed as

$$
\frac{\partial n}{\partial t}+\nabla_{\mathbf{x}} \cdot(\overline{\mathbf{v}} n)=-\frac{\partial}{\partial D}(R n)+\dot{Q}_{\mathrm{b}}+\dot{Q}_{\mathrm{c}}
$$

with $\dot{Q}_{\mathrm{b}}=\int \dot{Q}_{\mathrm{b}}^{\prime} \mathrm{dv}$ and $\dot{Q}_{\mathrm{c}}=\int \dot{Q}_{\mathrm{c}}^{\prime} \mathrm{d} \mathbf{v}$. In the absence of evaporation or dissolution, this becomes

$$
\frac{\partial n}{\partial t}+\nabla_{\mathbf{x}} \cdot(\overline{\mathbf{v}} n)=\dot{Q}_{\mathrm{b}}+\dot{Q}_{\mathrm{c}} .
$$

To close the problem, one needs reliable models for $\dot{Q}_{b}$ and $\dot{Q}_{c}$. Over the years, a considerable effort has been devoted to their modeling. In the turbine agitator experiments widely used in chemical engineering applications, $n$ and $\overline{\mathbf{v}}$ are assumed to be uniform throughout the vessel, and Eq. (4) simplifies to

$$
\frac{\partial n(D, t)}{\partial t}=\dot{Q}_{\mathrm{b}}(D)+\dot{Q}_{\mathrm{c}}(D) \text {. }
$$

In any other experimental conditions, including turbulent jet experiments, the contribution of the convective term, $\nabla_{\mathbf{x}} \cdot(\overline{\mathbf{v}} n)$, must be retained (see Martínez-Bazán et al., 1999a b). $\dot{Q}_{\mathrm{b}}$ is usually modeled as

$$
\dot{Q}_{\mathrm{b}}(D)=\int_{D}^{\infty} m\left(D_{0}\right) f\left(D, D_{0}\right) g\left(D_{0}\right) n\left(D_{0}, t\right) \mathrm{d} D_{0}-g(D) n(D, t) .
$$

where $g(D)$ is the break up frequency of particles of size $D, m\left(D_{0}\right)$ is the mean number of particles resulting from the break up of a mother particle of size $D_{0}$, and $f\left(D, D_{0}\right)$ is the size distribution of daughter particles formed from the breakage of a mother particle of size $D_{0}$. The first term on the right-hand side of Eq. (6) accounts for the rate of formation of particles of size $D$ from the breakup of particles larger than $D$. The second term accounts from the rate of break-up of particles of diameter $D$. Similarly, $\dot{Q}_{c}$ is commonly modeled as 


$$
\begin{aligned}
\dot{Q}_{\mathrm{c}}= & \int_{0}^{V} \lambda\left(V-V_{1}, V_{1}\right) h\left(V-V_{1}, V_{1}\right) n\left(V-V_{1}, t\right) n\left(V_{1}, t\right) \mathrm{d} V_{1} \\
& -n(V, t) \int_{0}^{\infty} \lambda\left(V, V_{1}\right) h\left(V, V_{1}\right) n\left(V_{1}, t\right) \mathrm{d} V_{1},
\end{aligned}
$$

where $h\left(V-V_{1}, V_{1}\right)$ is the collision frequency between two particles of volume $V-V_{1}$ and $V_{1}$, respectively, and $\lambda\left(V-V_{1}, V_{1}\right)$ is their collision efficiency. The terms on the right-hand side of Eq. (7) account for the formation and loss of particles of volume $V$ due to coalescence, respectively. Combining Eqs. (5)-(7), the population balance equation is written as

$$
\begin{aligned}
\frac{\partial n(D, t)}{\partial t}= & \int_{D}^{\infty} m\left(D_{0}\right) f\left(D, D_{0}\right) g\left(D_{0}\right) n\left(D_{0}, t\right) \mathrm{d} D_{0}-g(D) n(D, t) \\
& +\int_{0}^{V} \lambda\left(V-V_{1}, V_{1}\right) h\left(V-V_{1}, V_{1}\right) n\left(V-V_{1}, t\right) n\left(V_{1}, t\right) \mathrm{d} V_{1} \\
& -n(V, t) \int_{0}^{\infty} \lambda\left(V, V_{1}\right) h\left(V, V_{1}\right) n\left(V_{1}, t\right) \mathrm{d} V_{1} .
\end{aligned}
$$

The rate of change of the number density of particles, $\partial n(D, t) / \partial t$, can be expressed as a Birth rate and a Death rate of particles

$$
\frac{\partial n(D, t)}{\partial t}=B_{i}(D, t)-D_{\mathrm{e}}(D, t)
$$

where the Birth rate is

$$
\begin{aligned}
B_{i}(D, t)= & \int_{D}^{\infty} m\left(D_{0}\right) f\left(D, D_{0}\right) g\left(D_{0}\right) n\left(D_{0}, t\right) \mathrm{d} D_{0} \\
& +\int_{0}^{V} \lambda\left(V-V_{1}, V_{1}\right) h\left(V-V_{1}, V_{1}\right) n\left(V-V_{1}, t\right) n\left(V_{1}, t\right) \mathrm{d} V_{1}
\end{aligned}
$$

and the Death rate is,

$$
D_{e}(D, t)=g(D) n(D, t)+n(V, t) \int_{0}^{\infty} \lambda\left(V, V_{1}\right) h\left(V, V_{1}\right) n\left(V_{1}, t\right) \mathrm{d} V_{1} .
$$

Various models have been used to determine $g(D)$ and $f\left(D, D_{0}\right)$ in the break-up problem, and $h\left(V, V_{1}\right)$ and $\lambda\left(V, V_{1}\right)$ in the coalescence problem. We will focus our attention on the break-up, or fragmentation problem, and restrict our discussion to models for $g(D)$ and $f\left(D, D_{0}\right)$. A review of models for the closure parameters in the coalescence problem can be found in Kolev (1993) and elsewhere.

\section{Turbulent particle break-up models}

We consider systems in which the dispersed and continuous phases travel at the same mean velocity. Thus, aerodynamic fragmentation due to a mean velocity difference between the phases does not occur. Furthermore, we limit our discussion to systems in which the carrier phase 
turbulence can be regarded as locally homogeneous and isotropic. A review of aerodynamic break-up can be found in Kolev (1993) and in Lasheras and Hopfinger (2000). In the following, we present a comparative analysis of some of the more commonly used turbulent fragmentation models.

\subsection{Models for the particle break-up frequency, $g(D)$}

Coulaloglou and Tavlarides (1977) defined the break-up frequency of a particle of size $D$ as

$$
g(D)=\left(\frac{1}{\text { break-up time }}\right)\left(\frac{\text { fraction of }}{\text { drops breaking }}\right)=\frac{1}{t_{\mathrm{b}}} \frac{\Delta N(D)}{N(D)},
$$

where $N(D)$ is the total number of particles of size $D$. They modeled the fraction of drops breaking as

$$
\frac{\Delta N(D)}{N(D)}=\exp \left(-\frac{E_{c}}{\bar{E}}\right)
$$

with $E_{\mathrm{c}}=c_{1} \sigma D^{2}$ being the surface energy, $\bar{E}=c_{2} \rho D^{3} \overline{\Delta u^{2}}(D)$ a mean turbulent kinetic energy, and $\overline{\Delta u^{2}}(D)$ the mean squared value of the velocity fluctuations between two points separated by a distance $D$. If one is dealing with homogeneous and isotropic turbulence, and if $D$ is within the inertial subrange, this term can be written as (Batchelor, 1956)

$$
\overline{\Delta u^{2}}(D)=\overline{|u(x+D, t)-u(x, t)|^{2}}=c_{3} \epsilon^{2 / 3} D^{2 / 3} .
$$

Coulaloglou and Tavlarides assumed that the break-up time is given by the turbulent (eddy) turnover time,

$$
t_{\mathrm{b}} \propto D^{2 / 3} \epsilon^{-1 / 3}
$$

Substituting Eqs. (13)-(15) into Eq. (12) gives ${ }^{1}$

$$
g_{\mathrm{c}}(D)=C_{c 1} D^{-2 / 3} \epsilon^{1 / 3} \exp \left(-\frac{C_{c 2} \sigma}{\rho \epsilon^{2 / 3} D^{5 / 3}}\right),
$$

where $C_{c 1}$ and $C_{c 2}$ are two constants to be found experimentally.

Konno et al. (1980) used the basic formulation of Coulaloglou and Tavlarides to determine the particle break-up frequency,

$$
g(D)=C_{k} \frac{\sqrt{\overline{\Delta u^{2}}(D)}}{D} \int_{u_{\mathrm{c}}}^{\infty} P(u(D)) \mathrm{d} u(D) .
$$

However, Konno et al. represented the probability density distribution of relative velocity $u(D)$ by a Maxwell distribution 


$$
P(u(D))=4 \pi\left(\frac{3}{2 \pi \overline{\Delta u^{2}}(D)}\right)^{3 / 2} u^{2}(D) \exp \left(-\frac{3}{2} \frac{u^{2}(D)}{\overline{\Delta u^{2}}(D)}\right)
$$

resulting in a break-up frequency

$$
g_{k}(D)=C_{k} \frac{\sqrt{\overline{\Delta u^{2}}(D)}}{D} \int_{t^{*}}^{\infty} 3 \sqrt{\frac{6}{\pi}} x^{2} \exp \left(-\frac{3 x^{2}}{2}\right) \mathrm{d} x
$$

where the lower limit of integration is $u^{*}=u_{\mathrm{c}} / \sqrt{\Delta u^{2}}(D)$ and $u_{\mathrm{c}}$ is a critical velocity.

Following arguments from the kinetic theory of gases, Prince and Blanch (1990) postulated in their model that particle break-up is the result of collisions between particles and turbulent eddies. Their break-up frequency, therefore, is given by a collision rate multiplied by a break-up efficiency, $g(D)=\theta_{D e} F(u)$. They defined the collision rate as

$$
\theta_{D e}=n_{\mathrm{e}} S_{D \mathrm{e}}\left(\overline{\Delta u_{\mathrm{t} D}^{2}}+\overline{\Delta u_{\mathrm{te}}^{2}}\right)^{1 / 2},
$$

where $n_{\mathrm{e}}$ is the concentration of eddies in the size range of interest, $\overline{\Delta u_{\mathrm{t} D}^{2}}$ and $\overline{\Delta u_{\mathrm{te}}^{2}}$ are the average turbulent velocity of particles and eddies, respectively, and $S_{D e}$ is the collision cross-sectional area between particles of radius $D / 2$ and eddies of size $r_{\mathrm{e}}=\pi / k$, given by

$$
S_{D e}=\frac{\pi}{4}\left(D / 2+r_{\mathrm{e}}\right)^{2} .
$$

The number density of eddies within a given size range is obtained by integrating the energy spectrum,

$$
\frac{\mathrm{d} n_{\mathrm{e}}(k)}{\mathrm{d} k}=0.1 k^{2}
$$

Prince and Blanch point out that $\mathrm{Eq}$. (22) gives an infinite number of eddies as the wave number $k$ goes to infinity (small-size eddies). To avoid this problem, they arbitrarily chose a minimum eddy size equal to $20 \%$ of the particle diameter, arguing that eddies with a characteristic length equal to $20 \%$ of the drop diameter contain only $0.5 \%$ of the kinetic energy associated with an eddy of the size of the particle (this point will be further discussed later on). Prince and Blanch defined a break-up efficiency equal to that given by Coulaloglou and Tavlarides (1977),

$$
F(u)=\exp \left(-\frac{u_{c D}^{2}}{\overline{\Delta u_{\mathrm{te}}^{2}}}\right)
$$

where $^{2}$

$$
u_{c D}=1.52\left(\frac{\sigma}{\rho D}\right)^{1 / 2} .
$$

The break-up frequency is then given by 


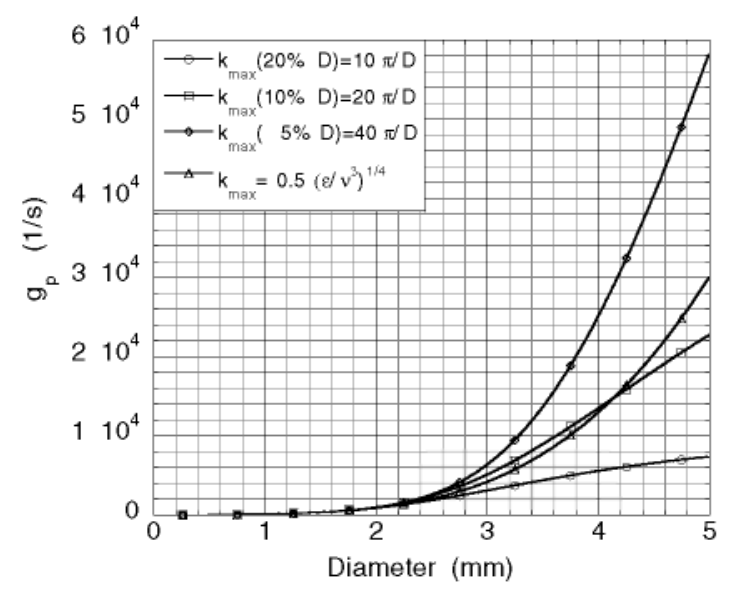

Fig. 1. Break-up frequency. Prince and Blanch (1990) model. $\sigma=0.072 \mathrm{~N} / \mathrm{m}^{-1}, \rho=1000 \mathrm{~kg} / \mathrm{m}^{3}, \epsilon=1 \mathrm{~m}^{2} / \mathrm{s}^{3}$.

$$
g_{p}\left(D_{i}\right)=\sum_{\mathrm{e}} n_{\mathrm{e}} S_{D \mathrm{e}}\left(\overline{\Delta u_{\mathrm{t} D}^{2}}+\overline{\Delta u_{\mathrm{te}}^{2}}\right)^{1 / 2} \exp \left(-\frac{u_{c D}^{2}}{\overline{\Delta u_{\mathrm{te}}^{2}}}\right),
$$

with $\sqrt{\overline{\Delta u_{\mathrm{t}}^{2}}}=1.4(\epsilon D)^{1 / 3}$. In integral form, the break-up frequency can be written as

$$
\begin{aligned}
g_{p}(D)= & \int_{0}^{10 \pi / D} \frac{0.14 \pi}{16}\left(D+\frac{2 \pi}{k}\right)^{2}\left(D^{2 / 3}+\left(\frac{2 \pi}{k}\right)^{2 / 3}\right)^{1 / 2} \epsilon^{1 / 3} \\
& \times \exp \left[-\frac{1.18}{(2 \pi)^{2 / 3}} \frac{\sigma k^{2 / 3}}{\rho D \epsilon^{2 / 3}}\right] k^{2} \mathrm{~d} k .
\end{aligned}
$$

Prince and Blanch did not define the lower limit of integration in Eq. (26), and arbitrarily took $k_{\max }=2 \pi / 0.2 D$ to be the maximum wave number. Although they claimed that eddies with lengths less than $20 \%$ of the particle diameter do not have enough energy to break up the particle, one can show that their model is very sensitive to the upper limit of integration, and therefore, it cannot be chosen arbitrarily. In Fig. 1, we show the break-up frequency obtained using the Prince and Blanch model for different values of the upper limit of integration. We varied the maximum wave number from that corresponding to $20 \%$ of the particle diameter $\left(k_{\max }=10 \pi / D\right)$ to that corresponding to $5 \%$ of the particle diameter $\left(k_{\max }=40 \pi / D\right)$. In addition, we have also shown the break-up frequency obtained by integrating Eq. (26) to the maximum wave number, corresponding to the Kolmogorov scale, defined as $k_{\eta}=0.5 \epsilon^{1 / 4} / v^{3 / 4}$. Contrary to their assumptions, Fig. 1 shows that the break-up frequency given by the Prince and Blanch model is, in fact, highly dependent on the limits of integration, and therefore, they must be clearly specified.

All of the above-described models are functions of empirically determined constants. Figs. 2(a) and (b) show the diameter dependence of the break-up frequency, calculated using the Coulaloglou and Tavlarides, Konno et al., and Prince and Blanch models for the cases of $\epsilon=1 \mathrm{~m}^{2} / \mathrm{s}^{3}$ and $\epsilon=1000 \mathrm{~m}^{2} / \mathrm{s}^{3}$, respectively. To compare the different models on one plot, we have normalized the break-up frequency determined from each model by its maximum value. The models 

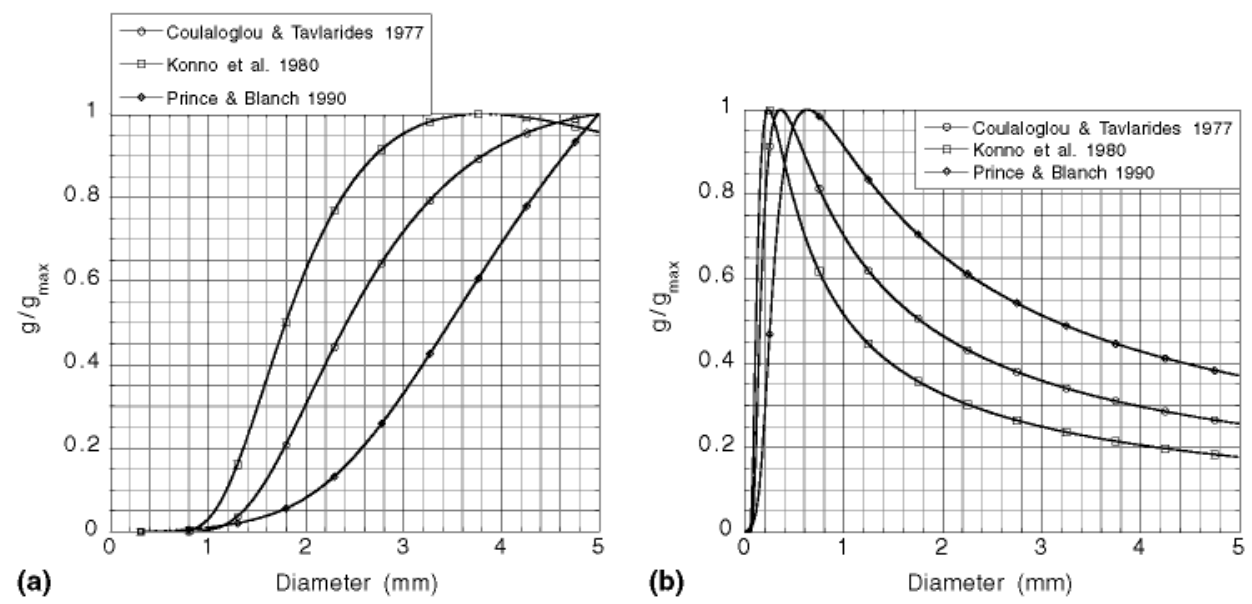

Fig. 2. Comparison of various break-up frequency models. (a) $\sigma=0.072 \mathrm{~N} / \mathrm{m}^{-1}, \rho=1000 \mathrm{~kg} / \mathrm{m}^{3}, \epsilon=1 \mathrm{~m}^{2} / \mathrm{s}^{3}$. (b) $\sigma=0.072 \mathrm{~N} / \mathrm{m}^{-1}, \rho=1000 \mathrm{~kg} / \mathrm{m}^{3}, \epsilon=1000 \mathrm{~m}^{2} / \mathrm{s}^{3}$.

proposed by Coulaloglou and Tavlarides and Konno et al. show a maximum in the break-up frequency as the drop diameter increases. This fact is not as evident in the model proposed by Prince and Blanch for low values of $\epsilon$, in which case, it seems that the break-up frequency increases monotonically with the drop diameter. On the other hand, Fig. 2(b) shows that, at higher values of $\epsilon$, the Prince and Blanch model also goes through a maximum as the drop diameter increases. In these plots, the upper limit of integration was taken to be equal to the wave number associated with an eddy of length equal to $20 \%$ of the drop diameter.

Tsouris and Tavlarides (1994) criticized their original model because it predicted a critical diameter whose break-up frequency is maximized. As shown in Fig. 2(b), the Prince and Blanch and Konno models exhibit this same behavior. Tsouris and Tavlarides considered this nonmonotonic behavior to be erroneous, and proposed a new model which predicted a monotonic increase of the break-up frequency with the drop diameter. Their new model was based on a particle-eddy collision model slightly different from that proposed by Prince and Blanch,

$$
g_{\mathrm{t}}(D)=C_{\mathrm{t} 1} \int_{n_{\mathrm{e}}} S_{D \mathrm{e}}\left(\overline{\Delta u_{\mathrm{te}}^{2}}+\overline{\Delta u_{\mathrm{tD}}^{2}}\right)^{1 / 2} \exp \left[-\frac{C_{\mathrm{t} 2} E_{\mathrm{c}}}{e}\right] \mathrm{d} n_{\mathrm{e}},
$$

where $S_{D \mathrm{e}}=\pi\left(D_{\mathrm{e}}+D\right)^{2}$ is the cross-section area, and $\overline{\Delta u_{\mathrm{t} D}^{2}}=1.07 \epsilon^{2 / 3} D^{2 / 3}$ and $\overline{\Delta u_{\mathrm{te}}^{2}}=8.2 \epsilon^{2 / 3} k^{-2 / 3}$ are the average turbulent velocities of a particle of diameter $D$ and a turbulent eddy of length $D_{\mathrm{e}}=2 / k$, respectively. The average energy of an eddy of size $D_{\mathrm{e}}$ was defined as

$$
e=\frac{1}{2} \frac{\rho \pi D_{\mathrm{e}}^{3}}{6} 8.2 \epsilon^{2 / 3}\left(\frac{D_{\mathrm{e}}}{2}\right)^{2 / 3}=0.43 \rho \pi D_{\mathrm{e}}^{11 / 3} \epsilon^{2 / 3} .
$$

The main difference between the Tsouris and Tavlarides model and the Prince and Blanch model is the value of the activation energy used. Tsouris and Tavlarides proposed an expression for the minimum energy needed for break-up to occur given by the average excess of surface energy needed to form a pair made up of a small particle and a large one as compared to forming two particles of the same volume. Stated mathematically, this gives 


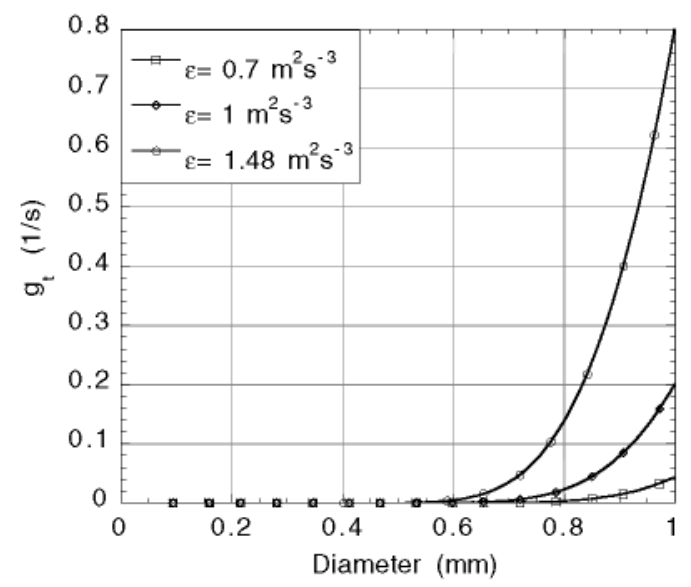

Fig. 3. Break-up frequency. Tsouris and Tavlarides (1994) model. $\sigma=0.072 \mathrm{~N} / \mathrm{m}^{-1}, \rho=1000 \mathrm{~kg} / \mathrm{m}^{3}$.

$$
E_{\mathrm{c}}=\frac{\pi \sigma}{2}\left[2\left(\frac{D}{2^{1 / 3}}\right)^{2}+D_{\max }^{2}+D_{\min }^{2}-2 D^{2}\right] .
$$

Here, $D_{\min }$ is a minimum drop size and $D_{\max }=\left(D^{3}-D_{\min }^{3}\right)^{1 / 3}$ is the diameter of a particle with complementary volume. Their expression for the break-up frequency is

$$
\begin{aligned}
g_{\mathrm{t}}(D)= & C_{\mathrm{t} 1} F(\phi) \epsilon^{1 / 3} \int_{2 / D}^{2 / D_{\min , e}} k^{2}\left(D+\frac{2}{k}\right)^{2}\left(1.07 D^{2 / 3}+\frac{8.2}{k^{2 / 3}}\right)^{1 / 2} \\
& \times \exp \left[-\frac{C_{\mathrm{t} 2} \pi \sigma}{2} \frac{\left[2\left(\frac{D}{2^{1 / 3}}\right)^{2}+D_{\max }^{2}+D_{\min }^{2}-2 D^{2}\right]}{0.43 \rho \pi(2 / k)^{11 / 3} \epsilon^{2 / 3}}\right] \mathrm{d} k,
\end{aligned}
$$

where $F(\phi)$ is a turbulence damping factor due to the presence of the disperse phase, $\phi$ is the volume fraction of the disperse phase, and $D_{\text {min,e }}$ is an arbitrarily defined minimum eddy size. Arguing that eddy sizes larger than the particle diameter transport the particle but do not affect its break-up and that eddies of size smaller than $D_{\text {min,e }}$ do not have enough energy to break the particle, Tsouris and Tavlarides proposed to integrate Eq. (30) from wave number $k_{\min }=2 / D$ (of the order of the particle size) to $k_{\max }=2 / D_{\text {min,e }}$. Based on their arguments, the break-up frequency of a particle whose diameter is within the inertial subrange $\left(L_{\mathrm{e}}>D>\eta\right)$ should be independent of the limits of integration as long as $2 / L_{\mathrm{e}}<k_{\min }<2 / D$ and $2 / \eta>k_{\max }>2 / D_{\min , \mathrm{e}}$, where $L_{\mathrm{e}}$ is the integral scale and $\eta$ is the Kolmogorov scale. Surprisingly, this argument does not hold true in Eq. (30). Notice that if the lower limit of integration is taken as $2 / L_{\mathrm{e}}$ instead of $2 / D$, the break-up frequency of small particles is considerably higher. In this case, the model gives a decreasing dependence of the break-up frequency with the particle diameter.

Fig. 3 shows the dependence of the break-up frequency on the particle diameter for different values of $\epsilon$. This figure, similar to Fig. 1(a) in Tsouris and Tavlarides (1994), was calculated by integrating Eq. (30) from $k_{\min }=2 / D$ to $k_{\max }=2 / D_{\min , \mathrm{e}}$. The break-up frequency obtained increases continuously with the particle diameter. The effect of the chosen lower limit of integration 


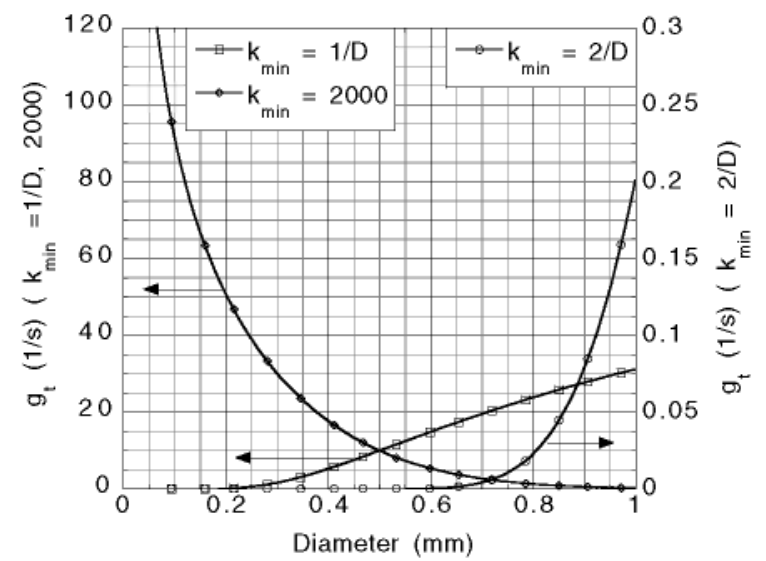

Fig. 4. Break-up frequency. Tsouris and Tavlarides (1994) model. $\sigma=0.072 \mathrm{~N} / \mathrm{m}^{-1}, \rho=1000 \mathrm{~kg} / \mathrm{m}^{3}, \epsilon=1 \mathrm{~m}^{2} / \mathrm{s}^{3}$.

on the break-up frequency given by the model is shown in Fig. 4. Note that when $k_{\min }$ is changed from $k_{\min }=2 / D$ to $k_{\min }=1 / D$ the break-up frequency of particles of $1 \mathrm{~mm}$ diameter changes from $0.2 \mathrm{~s}^{-1}$ to $32 \mathrm{~s}^{-1}$. Also note that when the lower limit is chosen to be $k_{\min }=2 / L_{\mathrm{e}}$ with $L_{\mathrm{e}}=1$ $\mathrm{mm}$ (the maximum drop size), the break-up frequency decreases with the particle diameter. These results clearly disagree with the premise that eddies larger than the particle diameter do not contribute to its break-up, as assumed by the model.

Luo and Svendsen (1996) also proposed a kinetic theory-type model, where the break-up frequency is calculated as a collision frequency between eddies and particles multiplied by a collision efficiency, $g(D)=\theta_{D \mathrm{e}} F(D)$. In their case, the collision frequency of eddies of size between $D_{\mathrm{e}}$ and $D_{\mathrm{e}}+\mathrm{d} D_{\mathrm{e}}$ with a particle of size $D$ is written as

$$
\theta_{D \mathrm{e}}(D)=\frac{\pi}{4}\left(D+D_{\mathrm{e}}\right)^{2} \frac{u_{D_{\mathrm{e}}}^{2}}{1 / 2} \frac{\mathrm{d} n_{\mathrm{e}}}{\mathrm{d} D_{\mathrm{e}}},
$$

with $\overline{\Delta u_{D_{\mathrm{e}}}^{2}}=\beta^{1 / 2}\left(\epsilon D_{\mathrm{e}}\right)^{1 / 3}$, and $\beta=2.045$. The number density of eddies, $n_{\mathrm{e}}$, was defined as

$$
\frac{\mathrm{d} n_{\mathrm{e}}}{\mathrm{d} D_{\mathrm{e}}}=\frac{0.822(1-\phi)}{D_{\mathrm{e}}^{4}},
$$

where $\phi$ is the volume fraction of dispersed phase. In dimensionless variables,

$$
\theta_{D \mathrm{e}}(\xi)=0.923(1-\phi)(\epsilon D)^{1 / 3} \frac{(1+\xi)^{2}}{D^{2} \xi^{11 / 3}},
$$

where $\xi=D_{\mathrm{e}} / D$. For the break-up efficiency, they proposed a function given by

$$
F(D)=\exp \left(-\frac{\bar{E}_{\mathrm{c}}(D)}{\bar{e}\left(D_{\mathrm{e}}\right)}\right),
$$

where $\bar{e}\left(D_{\mathrm{e}}\right)$ is the mean kinetic energy of an eddy of size $D_{\mathrm{e}}$, 


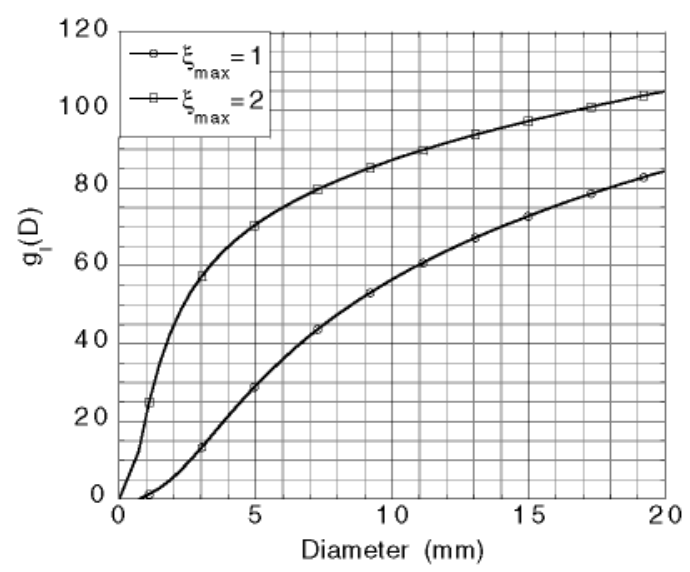

Fig. 5. Break-up frequency. Luo and Svendsen (1996) model. $\epsilon=1 \mathrm{~m}^{2} / \mathrm{s}^{3}, \sigma=0.072 \mathrm{~N} / \mathrm{m}^{-1}, \rho=1000 \mathrm{~kg} / \mathrm{m}^{3}$.

$$
\bar{e}\left(D_{\mathrm{e}}\right)=\rho \frac{\pi}{6} D_{\mathrm{e}}^{3} \frac{\overline{\Delta u_{D_{\mathrm{e}}}^{2}}}{2}=\frac{\pi \rho \beta}{12} D_{\mathrm{e}}^{11 / 3} \epsilon^{2 / 3}=\frac{\pi \rho \beta}{12} \xi^{11 / 3} D^{3}(D \epsilon)^{2 / 3},
$$

and $\bar{E}_{\mathrm{c}}(D)$ is the increase in surface energy when a particle of diameter $D$ is broken into two particles of size $D_{1}$ and $\left(D^{3}-D_{1}^{3}\right)^{1 / 3}$, respectively,

$$
\bar{E}_{\mathrm{c}}(D)=\pi \sigma\left(D_{1}^{2}+\left(D^{3}-D_{1}^{3}\right)^{2 / 3}-D^{2}\right)=\pi \sigma D^{2}\left(f_{V}^{2 / 3}+\left(1-f_{V}\right)^{2 / 3}-1\right)=C_{f} \pi \sigma D^{2},
$$

with $f_{V}=D_{1}^{3} / D^{3}$ and $0 \leqslant C_{f}=f_{V}^{2 / 3}+\left(1-f_{V}\right)^{2 / 3}-1 \leqslant 0.26$ depending on the daughter drop diameter. Combining Eqs. (31), (35) and (36), the break-up frequency of a particle of size $D$ that breaks into particles of sizes $D_{1}$ and $\left(D^{3}-D_{1}^{3}\right)^{1 / 3}$ is given by

$$
g_{l}\left(D ; D_{1}\right)=0.923(1-\phi)\left(\frac{\epsilon}{D^{2}}\right)^{1 / 3} \int_{\xi_{\min }}^{1} \frac{(1+\xi)^{2}}{\xi^{11 / 3}} \exp \left(-\frac{12 C_{f} \sigma}{\beta \rho \epsilon^{2 / 3} D^{5 / 3} \xi^{11 / 3}}\right) \mathrm{d} \xi,
$$

where $\xi_{\min }=D_{\min , \mathrm{e}} / D, D_{\min , \mathrm{e}} / \eta \approx 11.4-31.4$ and $\eta$ is the Kolmogorov microscale. The global break-up frequency of particles of size $D$ is calculated as

$$
g_{l}(D)=\frac{1}{2} \int_{0}^{1} g_{l}\left(D ; D_{1}\right) \mathrm{d} f_{V} .
$$

Luo and Svendsen argued that their model does not include any unknown, empirical parameters; however, their model does depend on the lower and upper limits of integration in Eq. (37), $\xi_{\text {min }}$ and 1, respectively. The selection of 1 as the upper limit indicates that Eq. (37) is integrated from the viscous scale to a length scale equal to the diameter of the particle. This model, like the previous models, is very sensitive to the upper limit of integration. The values for the break-up frequency obtained from Eq. (37) is integrated over the entire inertial subrange compared to when it is integrated only up to $\xi=1$. This fact is apparent in Fig. 5 where we have calculated the breakup frequency of air bubbles in water for a large range of bubble diameters (comparable to Fig. 5 in Luo and Svendsen, 1996) for two different cases. The first case was obtained integrating Eq. (37) 
up to a length scale equal to the diameter of the particle, $\xi=1$. In the second case, the upper limit of the integral was extended up to a scale that was twice the size of the particle, $\xi=2$.

In an attempt to overcome the inconsistencies found in the above-described turbulent break-up models, Martínez-Bazán et al. (1999a) proposed a model based on purely kinematic ideas. Although their model was developed for the particular case of the break-up of air bubbles immersed in a turbulent water flow, it has been extended to the more general case of liquid-liquid systems (Eastwood et al., 2000). The basic premise of this model is that for a particle to break, its surface has to be deformed, and further, that this deformation energy must be provided by the turbulent stresses produced by the surrounding fluid.

The minimum energy needed to deform a particle of size $D$ is its surface energy,

$$
E_{\mathrm{s}}(D)=\pi \sigma D^{2} \text {. }
$$

If viscous forces can be neglected in comparison with surface tension forces, the confinement stress is ( $E_{s} /$ volume)

$$
\tau_{\mathrm{s}}(D)=\frac{6 E_{\mathrm{s}}}{\pi D^{3}}=6 \frac{\sigma}{D} .
$$

If the size of these particles is within the inertial subrange, the average deformation stress, which results from velocity fluctuations existing in the liquid between two points separated by a distance $D$, can be estimated as

$$
\tau_{\mathrm{t}}(D)=\frac{1}{2} \rho \overline{\Delta u^{2}}(D)
$$

where $\overline{\Delta u^{2}}(D)$ is the mean value of the velocity fluctuations between two points separated by a characteristic distance $D$, and $\rho$ is the density of the continuous phase.

When the turbulent stresses are equal to the confining stresses, $\tau_{t}(D)=\tau_{s}(D)$, a critical diameter, $D_{\mathrm{c}}$, is defined such that particles with $D<D_{\mathrm{c}}$ are stable and will never break (Kolmogorov, 1949; Hinze, 1955). A particle of size $D>D_{\mathrm{c}}$ has a surface energy smaller than the deformation energy $\left(\tau_{\mathrm{s}}(D)<\tau_{\mathrm{t}}(D)\right)$, and thus, the particle deforms and eventually breaks up in a time $t_{\mathrm{b}}$. In a homogeneous and isotropic turbulent flow, one can apply Kolmogorov's universal theory to estimate $\overline{\Delta u^{2}}(D)$ as

$$
\overline{\Delta u^{2}}(D)=\overline{|u(x+D, t)-u(x, t)|^{2}}=\beta(\epsilon D)^{2 / 3},
$$

when $D$ is within the inertial subrange, $L_{\mathrm{e}}>D>\eta$. Eq. (42) is obtained by integrating from the Kolmogorov scale, $\eta$, to the integral scale, $L_{\mathrm{e}}$, as shown by Batchelor (Batchelor, 1956 ).

The critical diameter, $D_{\mathrm{c}}=(12 \sigma /(\beta \rho))^{3 / 5} \epsilon^{-2 / 5}$, is defined by the crossing point of the two curves shown in Fig. 6. Similar to any mechanical process (Newton's Law), Martínez-Bazán et al. postulate that the acceleration of the particle interface during deformation is proportional to the difference between the deformation and confinement forces acting on it. In other words, the probability of breaking a particle of size $D$ in time $t_{\mathrm{b}}$ increases as the difference between the pressure produced by the turbulent fluctuations on the surface of the particle, $\frac{1}{2} \rho \triangle u^{2}(D)$, and the restoring pressures caused by surface tension, $6 \sigma / D$, increases. On the other hand, the breakup frequency should decrease to a zero limit value as this difference of pressures vanishes. Thus, the particle break-up time can be estimated as 


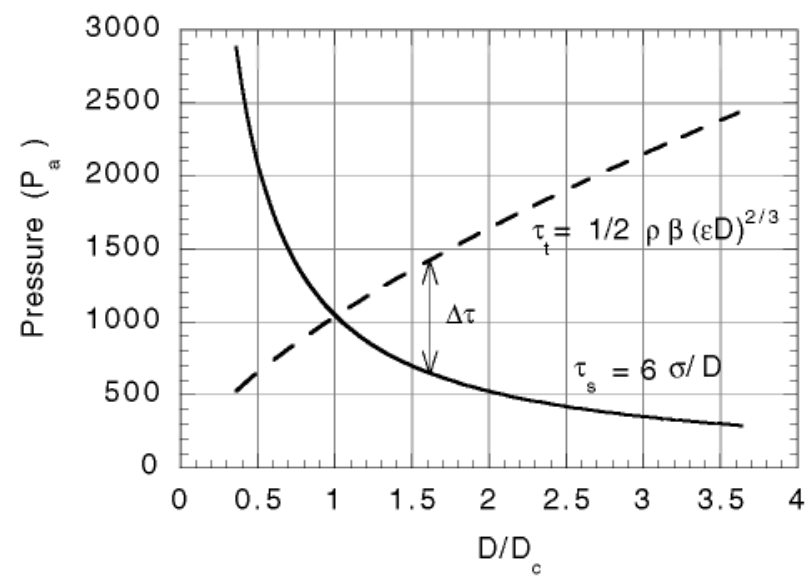

Fig. 6. Confining and turbulent stresses acting on a particle. Solid line is the confining pressure provided by surface tension and the broken line shows the deforming, turbulent stresses.

$$
t_{\mathrm{b}} \propto \frac{D}{u_{\mathrm{b}}}=\frac{D}{\sqrt{\overline{\Delta u^{2}}(D)-12 \frac{\sigma}{\rho D}}},
$$

where $u_{\mathrm{b}}$ is the characteristic velocity of the particle break-up process. The break-up frequency $g(\epsilon, D)$ is given by

$$
g(\epsilon, D)=\frac{1}{t_{\mathrm{b}}}=K_{\mathrm{g}} \frac{\sqrt{\overline{\Delta u^{2}}(D)-12 \frac{\sigma}{\rho D}}}{D}=K_{\mathrm{g}} \frac{\sqrt{\beta(\epsilon D)^{2 / 3}-12 \frac{\sigma}{\rho D}}}{D},
$$

where the constant $\beta=8.2$ was given by Batchelor (1956), and $K_{g}=0.25$ was found experimentally for the case of air bubbles in water.

The dependence of the break-up frequency, given by Eq. (44), on the particle diameter is shown in Fig. 7. The break-up frequency is zero for particles of size $D \leqslant D_{\mathrm{c}}$, and it increases rapidly for particles larger than the critical diameter, $D>D_{\mathrm{c}}$. It is important to note that after reaching a maximum at $D_{g_{\max }}=(9 / 4)^{3 / 5} D_{\mathrm{c}} \approx 1.63 D_{\mathrm{c}}$, the break-up frequency decreases monotonically with the particle size. The maximum break-up frequency, achieved at $D_{g_{\max }}$, is given by

$$
g_{\max }(\epsilon)=0.538 K_{g} \beta^{1 / 2} \epsilon^{3 / 5}\left(\frac{12 \sigma}{\rho \beta}\right)^{-2 / 5} .
$$

In summary, break-up frequency models based on arguments similar to the kinetic theory of gases assume that turbulence is manifested as an array of eddies with well-defined sizes and number densities. They require the definition of a collision cross-section and an eddy arrival frequency based on this assumption. They also require the specification of closure parameters (e.g. limits of integration) that can significantly alter their behavior. Some of the models for $g(D)$ discussed above are compared with each other and with experimental data obtained from the break-up of air bubbles immersed in a high Reynolds number, submerged water jet in Section 3 (for further comparisons, see Martínez-Bazán et al. (1999a,b)). 


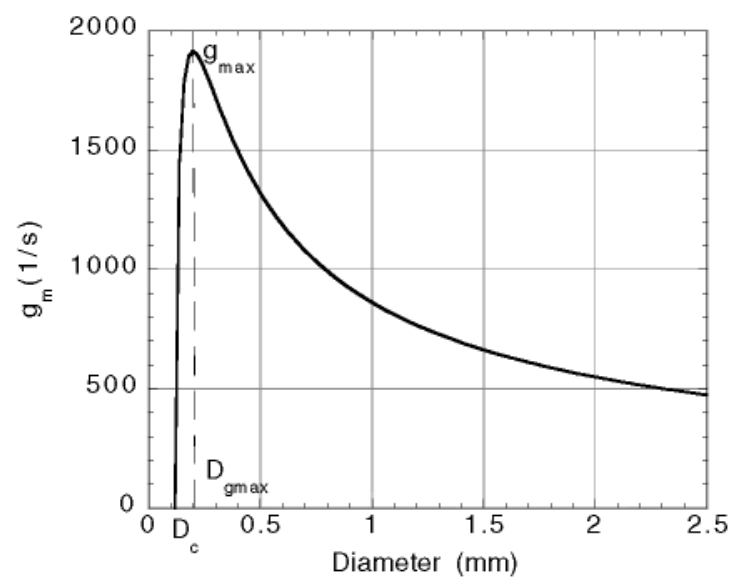

Fig. 7. Evolution of the particle break-up frequency with the particle diameter.

\subsection{Models for the daughter particle size distribution, $f\left(D, D_{0}\right)$}

As was shown in Eq. (6), a complete particle break-up model must include an expression for the size distribution of the daughter particles resulting from break-up of a mother particle of size $D_{0}$, $f\left(D, D_{0}\right)$, in addition to a model for $g(D)$. Historically, there have been three predominant approaches to the formulation of this term: statistical models, phenomenological models based on the change in surface energy of a breaking particle, and hybrid models which are based on a combination of both. Surface energy models have taken two forms: those based on the probability of sufficiently energetic eddy collisions, and those based on a balance of stresses existing at the particle surface. In all cases, both the shape of the daughter probability density function (pdf) and the number of daughter particles formed by a break-up event, $m\left(D_{0}\right)$, must be determined. The latter is done by either assuming a given number of daughter particles a priori, or by deriving an empirical relation for the number of daughter particles from available experimental data. A wide variety of approaches have been taken to determine the shape of the daughter particle pdf. Some of the more widely used models are discussed below.

\subsubsection{Statistical models}

One of the earliest statistical models for the daughter particle size distribution is that proposed by Valentas et al. (1966). Valentas considers two possible daughter particle pdfs. The first is a discrete model, in which a parent particle of diameter $D_{0}$ is assumed to split into equally-sized daughter particles of diameter $D_{0} / \mathrm{m}^{1 / 3}$, where $m$ is the number of daughter particles formed. For binary break-up $(m=2)$, this means that

$$
f\left(D, D_{0}\right)=\delta\left(D-\frac{D_{0}}{2^{1 / 3}}\right) .
$$

The use of a $\delta$-function pdf suggests that particle break-up is deterministic. Binary break-up into two equally sized daughter particles implies that at steady-state, no particles smaller than 
$D_{\max } / 2^{1 / 3}$ exist, where $D_{\max }$ is the largest stable drop diameter (Kolmogorov, 1949; Hinze, 1955). This result has never been confirmed by experiments (Nambiar et al., 1992).

The second model proposed by Valentas et al. is a logical, continuous analog of the discrete daughter particle pdf. In this case, it is assumed that the daughter particle sizes, for a parent particle of diameter $D_{0}$, are normally distributed about a mean value, $\bar{D}=D_{0} / m\left(D_{0}\right)^{1 / 3}$. Thus, $f\left(D, D_{0}\right)$ is given by

$$
f\left(D, D_{0}\right)=\frac{1}{\sigma \sqrt{2 \pi}} \exp \left[-\frac{(D-\bar{D})^{2}}{2 \sigma^{2}}\right] .
$$

Note that the normal distribution extends from $-\infty$ to $+\infty$. If $D$ must lie within a specified tolerance, $c$, of $\bar{D}$, such that $\bar{D}-c \leqslant D \leqslant \bar{D}+c$, then the variance, $\sigma$, must be defined as

$$
\sigma=\frac{D_{0}}{c m\left(D_{0}\right)^{1 / 3}},
$$

where $m$ is a function of $D_{0}$. It represents the average number of daughter particles formed by the break-up of a mother particle of a given size. In order to use this model, $m$ must be found from experimental data. Since $m$ is dependent on the flow conditions, it will vary from system to system. Valentas found that the results of his model were strongly dependent on the choice of $m$.

Coulaloglou and Tavlarides (1977) adopted the Valentas model in their work. They assumed binary break-up $(m=2)$ and fixed $\sigma$ such that greater than $99.6 \%$ of the particles formed were within the volume range 0 to $\pi D_{0}^{3} / 6(c=3)$. This gives the daughter particle size distribution as

$$
f\left(D^{3}, D_{0}^{3}\right)=\frac{2.4}{D_{0}^{3}} \exp \left[-\frac{4.5\left(2 D^{3}-D_{0}^{3}\right)^{2}}{D_{0}^{6}}\right] .
$$

Other authors, including Chatzi et al. (1989) and Chatzi and Kiparissides (1992) have employed the truncated normal function as a daughter particle size distribution. This is a statistical model, applying on average to a large population of particles. If it is argued that the break-up of a collection of particles is a composite of several independent, random events, then the normal distribution can be arrived at statistically; however, there is no physical justification for this model. The properties of the turbulent flow, and of the dispersed phase, enter into the model only through the choice of $m$. As the system and the level of underlying turbulence vary, the value of $m$ will vary. The appropriate value of $m$ can only be determined if experimental data is available.

Hsia and Tavlarides (1983) found that the truncated normal model proposed by Coulaloglou and Tavlarides (1977) was unable to predict the results obtained by Ross (1971), Verhoff et al. (1977), and Ross et al. (1978). Consequently, they modified the earlier work, assuming binary break-up and a beta distribution, rather than the truncated normal distribution. The particular beta distribution selected by Hsia and Tavlarides for their daughter particle pdf is given by

$$
f\left(D^{3}, D_{0}^{3}\right)=\frac{30}{D_{0}^{3}}\left(\frac{D^{3}}{D_{0}^{3}}\right)^{2}\left[1-\frac{D^{3}}{D_{0}^{3}}\right]^{2} .
$$

In a later work, Lee et al. (1987) assumed that the daughter particle pdf was a beta distribution of the form 


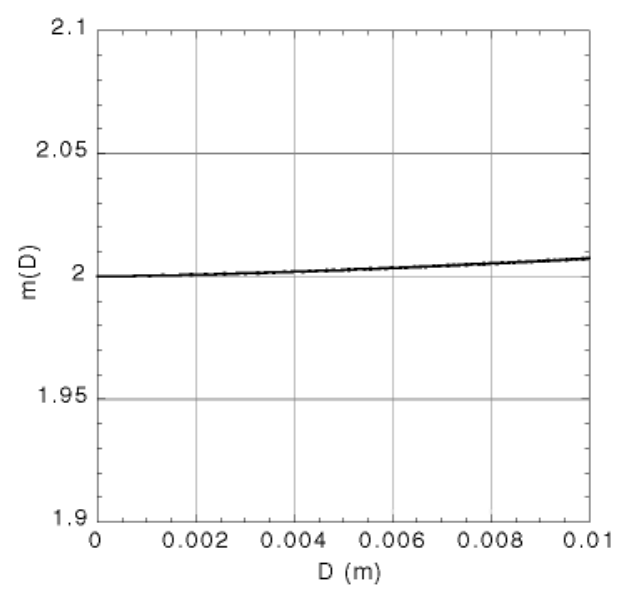

Fig. 8. Evolution of $m\left(v^{\prime}\right)$ with mother particle diameter for the Lee et al. (1987) model.

$$
f\left(v, v^{\prime}\right)=\frac{\Gamma(a+b)}{\Gamma(a) \Gamma(b) v^{\prime}}\left(\frac{v}{v^{\prime}}\right)^{a-1}\left[1-\frac{v}{v^{\prime}}\right]^{b-1},
$$

where $v^{\prime}$ is the volume of the mother particle and $f\left(v, v^{\prime}\right) \mathrm{d} v$ represents the fractional number of particles with volumes between $v$ and $v+\mathrm{d} v$ formed when a particle of volume $v^{\prime}$ breaks. $a$ and $b$ are empirically derived constants.

Lee et al. determined the values of $a$ and $b$ which best fit their experimental data (bubble breakup in an airlift column) by first assuming binary break-up, $\left(m\left(v^{\prime}\right)=2\right)$, and then assuming breakup of the form

$$
m\left(v^{\prime}\right)=2+c\left(v^{\prime}\right)^{n^{\prime}},
$$

where $c$ and $n^{\prime}$ are additional, empirically derived constants. For binary break-up $(c=0)$, the best value for both $a$ and $b$ was 2.0. The authors emphasize that the average number of particles formed per breakage event has a strong influence on the steady-state particle size distribution, and further, that their data was best-fit using the "multibreakage" (non-binary) model. They obtained the best fit to their data with $c=10.0$ and $n^{\prime}=0.5 . m\left(v^{\prime}\right)$ is plotted in Fig. 8 using these values. The authors state that bubble diameters in their system range from 1 to $8 \mathrm{~mm}$. Notice that $m\left(v^{\prime}\right)$ does not vary significantly from the binary breakage scenario $\left(m\left(v^{\prime}\right)=2.0\right)$ over this entire range. It is likely that Lee et al. obtain a better fit using the multibreakage model because, in this case, they also change additional parameters in their break-up frequency model.

The $\beta$-distribution is a two-parameter model that can assume a variety of shapes. Investigators have been able to fit a wider range of data with it than with the single parameter, truncated normal distribution. Like the truncated normal distribution, however, the proper selection of the adjustable parameters relies heavily on available experimental data. The values chosen for these parameters change both from apparatus-to-apparatus and with varying flow conditions. The truncated normal distribution employed by Coulaloglou and Tavlarides and the beta distributions used by Hsia and Tavlarides and by Lee et al. in their binary break-up model are shown in Fig. 9 . 


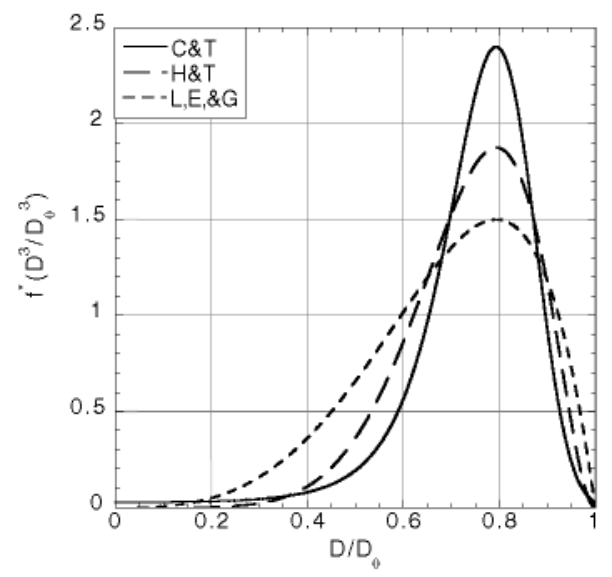

Fig. 9. Comparison of the non-dimensional daughter particle pdf models, $f^{*}\left(D^{3} / D_{0}^{3}\right)=D_{0}^{3} f\left(D^{3}, D_{0}^{3}\right)$ used by Coulaloglou and Tavlarides (1977) [C\&T], Hsia and Tavlarides (1983) [H\&T], and Lee et al. (1987) [L,E\&G]. For L,E,\&G, the curve shown is for binary breakage with $a=b=2.0 . D_{0}=3 \mathrm{~mm}$ for each model.

Collins and Knudsen (1970) investigated particle size distributions resulting from the break-up of an immiscible liquid in turbulent pipe flow. They compared a variety of daughter particle size distribution models, including the truncated normal distribution, with their experimental data. The best agreement was obtained by assuming that when a mother particle breaks, two daughter particles with uniformly distributed volume ratios are formed, along with a third, very small satellite particle. A uniform distribution with binary break-up was assumed by Randolph (1969) and also by Narsimhan et al. (1979).

Prince and Blanch (1990) derived a phenomenological model for particle break-up frequency based on surface energy considerations; however, they did not derive a companion model for the daughter particle size pdf. Rather, they assumed that a mother particle breaks into two daughter particles of random size. In this case, there is an equal probability for forming a daughter particle of any size. This gives a uniform distribution with binary break-up. There is no physical justification for selecting a uniform distribution model; however, it could be argued that at high levels of turbulence, an amount of energy sufficient to cause particle break-up might exist over a wide range of scales. This would cause a physically based daughter particle distribution to flatten-out at very high dissipation rates. Since turbulent fluctuations are not uniform over all scales, the uniform distribution remains an idealization, even at high dissipation rates of turbulent kinetic energy.

Hesketh et al. (1991) performed experiments to investigate particle break-up in turbulent pipe flow. They compared several different daughter particle size distributions, including: equal-volume breakage (e.g. the $\delta$-function model proposed by Valentas), random breakage (e.g. Prince and Blanch), attrition, and what the authors refer to as $1 / X$-type breakage. These models are shown in Fig. 10. $\beta\left(D_{0}^{3} ; D^{3}\right)$ is the probability density function for the formation of a daughter particle of diameter $D$ from a mother particle of diameter $D_{0}$, referred to in the current paper as $f\left(D, D_{0}\right) . f_{V}$ is the volume fraction, $D^{3} / D_{0}^{3}$. These authors found that their experimental data was best fit by employing a model that predicted daughter particle sizes somewhere between those predicted by 


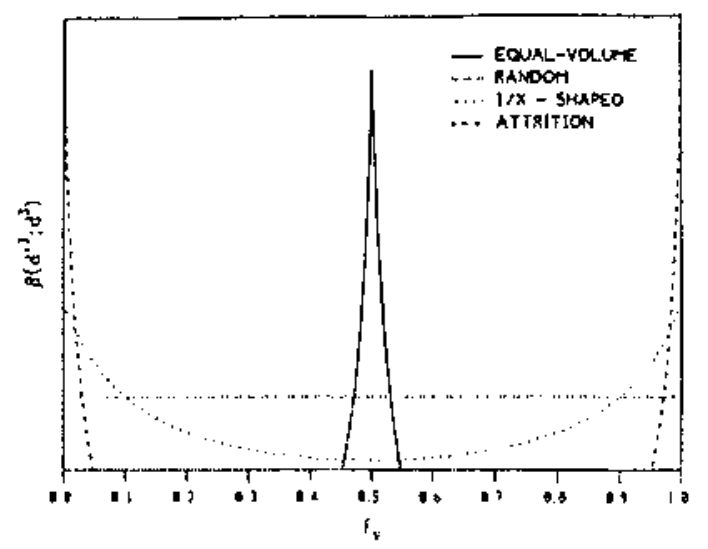

Fig. 10. (From Hesketh et al., 1991). Various statistical daughter particle pdf models. $\beta\left(D_{0}^{3} ; D^{3}\right)$ is the dimensional probability density function for the formation of a daughter particle of diameter $D$ from a mother particle of diameter $D_{0}, f_{V}$ is the volume fraction of the daughter particle.

random and attrition breakage. They found that random breakage over-predicted the formation of daughters with $f_{V}$ near 0.5 and therefore over-estimated the Sauter mean diameter, $D_{32}$, of the resulting particle distribution. On the other hand, attrition breakage over-predicted the formation of very small daughter particles and under-estimated $D_{32}$. The $1 / X$-type distribution used by these authors was selected based on a best-fit of the asymptotic value of $D_{32}$ for their system, and has the following form:

$$
\beta\left(D_{0}^{3} ; D^{3}\right)=\left[\frac{1}{\left(\left(D / D_{0}\right)^{3}+B\right)}+\frac{1}{\left(1-\left(D / D_{0}\right)^{3}+B\right)}-\frac{2}{(B+0.5)}\right] \frac{I}{D_{0}^{3}},
$$

where $B$ is an empirically determined constant and $I$ is a constant required for normalization. This model also requires an empirically determined estimate for the diameter, $D_{\min }$, of the smallest daughter particle formed. It should be noted that even when using the best fit obtained with the distribution given in Eq. (53), this model under-predicted the number of daughter particles seen in Hesketh's experiments with $0.225 \leqslant f_{V} \leqslant 0.5$. As with the other statistical models, the values of the empirical constants in this model will vary with different flow conditions, with initial particle size, and from apparatus-to-apparatus. In order to use the model, experimental data for the given conditions and apparatus must be available. This model has no physical justification; however, it has been used as a source of comparison for many of the surface-energy models described below.

More recent, purely statistical models have been proposed by Longuet-Higgins (1992) and Novikov and Dommermuth (1997). Longuet-Higgins simulated the break-up process by viewing it as a sequence of random divisions of a unit cube by a number of planes oriented parallel to the faces of the cube. He studied the size-pdf resulting from different numbers of cuts performed in one, two, and three dimensions. An infinite number of distributions are possible depending on the number of dimensions and on the number of cuts made. Longuet-Higgins showed that by adjusting the number of cuts performed in each dimension the model could match various 
experimental results. This model has the same shortcomings as those discussed above: it lacks physical justification and relies entirely on empirical data.

\subsubsection{Hybrid models}

Konno et al. (1980) proposed a statistical model that includes the distribution of energy among turbulent eddies of different scales. They assume that each mother particle, of volume $v_{0}$, is divided into $J$ equally-sized elements, such that $J=v_{0} / v_{\mathrm{e}}$, where $v_{\mathrm{e}}$ is the volume of a single element. They also assume that the number of daughter particles formed is equal to $m$ for each breakage event and that the volume of each daughter particle formed is an integral multiple of $v_{\mathrm{e}}$. Each daughter particle, specified by the index $i$, has a dimensionless volume given by $K_{i}=v_{i} / v_{\mathrm{e}}$. Since each breakage results in $m$ daughter particles, then, assuming an incompressible fluid, conservation of mass requires that all possible combinations of daughter particles satisfy the relationship

$$
K_{1}+K_{2}+K_{3}+\cdots+K_{m}=J .
$$

As $J$ and $m$ increase, the number of possible combinations of daughter particles increases rapidly (for instance, for $J=7$ and $m=3$, the number of possible combinations is 15). Konno et al. consider that the formation of daughter particles of given size results from interactions between the mother particle and turbulent eddies of that same size. The probability of forming a daughter particle of given size is then proportional to the kinetic energy contained in eddies of that length scale. If these eddies are independent of one another, then the probability of obtaining a combination of $K_{i}$ is given by their product

$$
P \propto E\left(K_{1}\right) E\left(K_{2}\right) E\left(K_{3}\right) \cdots E\left(K_{m}\right),
$$

where $E\left(K_{i}\right)$ is estimated from the following Heisenberg energy spectrum:

$$
E\left(K_{i}\right)=\left(\frac{8}{9 \alpha}\right)^{2 / 3} \epsilon^{2 / 3}\left(\frac{\pi}{6 v_{\mathrm{e}} K_{i}}\right)^{-5 / 3}\left[1+\frac{8 v_{\mathrm{e}}^{3}\left(\pi / 6 v_{\mathrm{e}} K_{i}\right)^{4}}{3 \alpha^{2}}\right]^{-4 / 3} .
$$

where $\alpha=0.51$.

In order to obtain a continuous distribution of daughter particles, $J$ must be large. In their analysis, the authors set $J=100$ and found the best fit with their experimental data by assuming that $m=3$. With these values of $J$ and $m$, Konno et al. (1983) show that the above model is wellapproximated by the following beta distribution:

$$
D_{0} f\left(D, D_{0}\right)=f^{*}\left(D / D_{0}\right)=\frac{\Gamma(12)}{\Gamma(3) \Gamma(9)}\left(\frac{D}{D_{0}}\right)^{8}\left(1-\frac{D}{D_{0}}\right)^{2} .
$$

This result is plotted in Fig. 11. Notice that this model predicts a high probability for the formation of equally sized daughter particles and a low probability for the formation of a very small daughter particle and its complement. Although the Heisenberg energy spectrum depends on the dissipation rate of turbulent kinetic energy, Konno's model is well-approximated by Eq. (57) which contains no dependence on the underlying turbulence. Also, as the size of the mother particle, $D_{0}$ is increased, the shape of $f^{*}\left(D / D_{0}\right)$ does not change. This behavior is contrary to available experimental observations. 


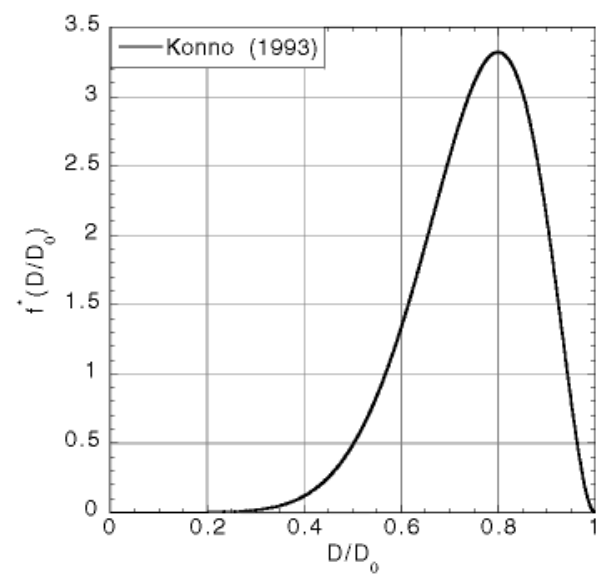

Fig. 11. Dimensionless daughter particle probability function predicted by Konno et al. (1980), as approximated by Konno et al. (1983).

\subsubsection{Surface energy models based on eddy collisions}

Ross and Curl (1973), Coulaloglou and Tavlarides (1977), Prince and Blanch (1990), and others, have argued that particle break-up should be a function of the difference in surface energy between a mother particle and the energy of a colliding eddy. Although these authors developed models for the particle break-up frequency, they relied upon statistical models for the resulting daughter particle size distribution. In the majority of cases, statistical models favor the formation of equally sized daughter particles (this is not true for the uniform distribution). This is at odds with the eddy collision-surface energy argument, however, since the energy required for collisioninduced breakage into two equally sized particles is greater than the energy required for break-up into a small and a large particle.

Nambiar et al. (1992) developed a phenomenological model for the breakage rate of viscous drops in stirred vessels. Their model differs from those listed in the previous paragraph in that in addition to a model for the break-up frequency, they also derived an expression for the daughter particle pdf. Nambiar et al. argue that particles break as a result of collisions with turbulent eddies of appropriate size and with sufficient energy. They postulate the existence of a minimum and maximum eddy size capable of particle breakage. The maximum eddy size capable of breaking a mother particle is given by the diameter of the particle itself. The possibility of finding eddies of length $D_{\mathrm{e}}$ smaller than a particle of diameter $D$ that are capable of causing breakage is dependent upon finding values of $D_{\mathrm{e}} \leqslant D$ that satisfy

$$
\frac{2}{\sqrt{\alpha}} \arctan \left(\frac{1}{2 \sqrt{\alpha}}\right) \leqslant\left(\frac{\operatorname{Re} G}{W e}\right)\left(\frac{D}{D_{\mathrm{I}}}\right)^{-1 / 3}\left(\frac{D_{\mathrm{e}}}{D}\right)^{2 / 3},
$$

where $R e$ is the Reynolds number, defined as $N D_{\mathrm{I}}^{2} \rho / \mu_{\mathrm{d}}$, We is the Weber number, defined as $\rho N^{2} D_{\mathrm{I}}^{3} / \sigma, N$ is the rotational speed of the impeller, and $D_{\mathrm{I}}$ is the impeller diameter. $G$ is related to the change in surface energy resulting from the formation of a daughter particle of volume $v$ from a mother particle of volume $v^{\prime}$ and is given by 


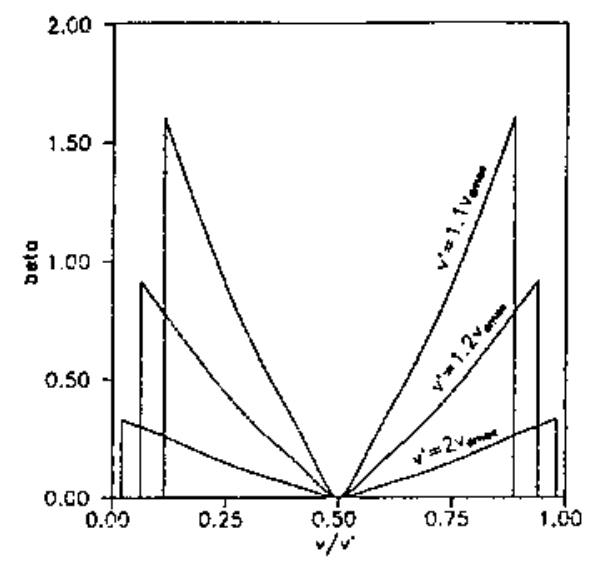

Fig. 12. (From Nambiar et al., 1992.) Dependence on the mother particle size for the dimensionless daughter particle pdf predicted by Nambiar et al. (1992). Beta is given by $v^{\prime} * f\left(v, v^{\prime}\right)$ in Eq. (62).

$$
G=\left(\frac{v^{\prime}-v}{v^{\prime}}\right)^{2 / 3}+\left(\frac{v}{v^{\prime}}\right)^{2 / 3} / 2^{1 / 3} .
$$

$\alpha$ is given by

$$
x=\frac{C\left(D_{\mathrm{e}} / D\right)^{2 / 3} W e}{G}\left(\frac{D}{D_{\mathrm{I}}}\right)^{5 / 3}-\frac{1}{4} .
$$

The constant $C$ relates the mean inertial stress, $\tau_{T}$, to the size and speed of the impeller and is determined from the relationship

$$
\bar{\tau}_{T}=C \rho N^{2} D_{\mathrm{I}}^{4 / 3} D_{\mathrm{e}}^{2 / 3} .
$$

In this model, $D_{\text {nin,e }}$ is found by solving Eq. (58) as a strict equality. $D_{\max }$ is found by solving this equality with $D_{\mathrm{c}} / D=1$. Particles with diameters less than $D_{\max }$ do not break. Particles with diameters equal to $D_{\max }$ undergo equal, binary breakage. The author argues that this is the case since these drops can be broken by eddies of only one size: those with diameters equal to the drop diameter. The symmetry of the problem predicates equal breakage. Particles larger than $D_{\max }$ are susceptible to asymmetric breakage. These particles have the following daughter particle size pdf:

$$
f\left(v, v^{\prime}\right)=\frac{4 \sin \left|\frac{\pi-2 \phi}{3}\right| f\left(D_{\mathrm{e}} \mid D_{\min , \mathrm{e}} \leqslant D_{\mathrm{e}} \leqslant D\right)}{\pi D_{\mathrm{c}} D \sin \phi},
$$

where $v$ is the volume of the daughter particle, $v^{\prime}$ is the volume of the mother particle, and $f\left(D_{\mathrm{e}} \mid D_{\text {min, } \mathrm{e}} \leqslant D_{\mathrm{e}} \leqslant D\right)$ is the conditional probability of finding an eddy of length $D_{\mathrm{e}}$ in the size range between $D_{\text {min,e }}$ and $D . \phi$ is determined from the equation

$$
\phi=\arccos \left(1-\frac{2 v}{v^{\prime}}\right) .
$$

Nambiar's daughter particle pdl is shown in Fig. 12. It has a V-shape and is symmetric about $v^{\prime} / 2$. There is zero probability of equal breakage and in increasing probability of breaking off either 
very large or very small daughter particles. The pdf reaches a maximum at its tails, $v_{\min }\left(v^{\prime}\right)$ and $v^{\prime}-v_{\min }\left(v^{\prime}\right)$. The smallest daughter particle size, $v_{\min }\left(v^{\prime}\right)$ is determined by $D_{\text {min,e }}\left(D_{0}\right)$, where $D_{0}$ is the diameter corresponding to $v^{\prime}$. The probability of breaking off a daughter particle smaller than $v_{\text {min }}\left(v^{\prime}\right)$ or larger than $v^{\prime}-v_{\min }\left(v^{\prime}\right)$ is zero. It is important to note that as the size of the mother particle increases, this model approaches the uniform distribution in that its tails become less pronounced. In other words, as $v^{\prime}$ increases, the relative probability of creating particles of approximately equal size increases; however, the probability of creating daughter particles of exactly the same size remains zero, unless the mother particle diameter is equal to $D_{\max }$. It is highly unlikely that equal breakage never takes place for particles with sizes other than $D_{\max }$.

One of the most popular phenomenological models for the daughter particle pdf is that proposed by Tsouris and Tavlarides (1994). Like Nambiar, their model assumes binary break-up $\left(m\left(D_{0}\right)=2\right)$. The authors postulate that the formation of a daughter particle of size $D_{1}$ is inversely proportional to the energy required to split a mother particle of size $D_{0}$ into a particle of size $D_{1}$ and its complementary particle of size $D_{2}$. This energy requirement is proportional to the excess surface area generated by splitting the mother particle

$$
e\left(D_{1}\right)=\pi \sigma D_{1}^{2}+\pi \sigma D_{2}^{2}-\pi \sigma D_{0}^{2}=\pi \sigma D_{0}^{2}\left[\left(\frac{D_{1}}{D_{0}}\right)^{2}+\left[1-\left(\frac{D_{1}}{D_{0}}\right)^{3}\right]^{2 / 3}-1\right] .
$$

This expression is maximized when two daughter particles of equal size $\left(D_{1}=D_{2}=D_{0} / 2^{1 / 3}\right)$ are formed, giving a maximum energy equal to

$$
e_{\max }=\pi \sigma D_{0}^{2}\left[2^{1 / 3}-1\right] .
$$

Eq. (64) is minimized when $D_{1}=0$, that is, when the mother particle does not break. In order to allow particles to break, a minimum diameter, $D_{\min }$ and its corresponding surface energy, $e_{\min }=$ $\pi \sigma D_{\min }^{2}$ should be specified. With these expressions for the maximum and minimum energies required to create daughter particles, the daughter particle size pdf is

$$
f\left(D_{1}, D_{0}\right)=\frac{e_{\min }+\left[e_{\max }-e\left(D_{1}\right)\right]}{\int_{D_{\min }}^{D_{0}} e_{\min }+\left[e_{\max }-e\left(D_{1}\right)\right] \mathrm{d} D_{1}} .
$$

This distribution has a U-shape, with a minimum probability for the formation of two equally sized daughter particles and a maximum probability for the formation of a very large daughter particle and its complement of size $D_{\min }$. A plot of this function is shown in Fig. 13. For this plot, $e_{\min }=0$; however, the distribution function does not go to infinity at its tails. In fact, the pdf approaches a constant value at each tail, suggesting that although the energy requirement for stripping off successively smaller daughter particles decreases, the probability of this event does not increase, but instead reaches a constant value. This result is contrary to the argument that the daughter particle size distribution is linearly related to the energy required for the formation of the daughter drops. Like Nambiar, this model predicts a zero probability for the formation of daughter particles of equal size (when $e_{\min }=0$ ). It is highly unlikely that equally sized daughter particles can be ruled out altogether. Furthermore, this model has no dependence on either the dissipation rate of turbulent kinetic energy or on the initial particle size. This behavior does not match experimental observation. 


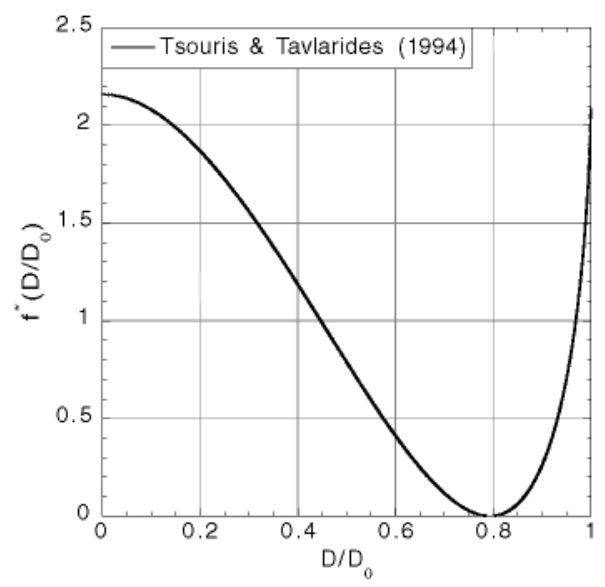

Fig. 13. Dimensionless daughter particle pdf predicted by Tsouris and Tavlarides (1994) model. $\sigma=0.072 \mathrm{~N} / \mathrm{m}$.

Luo and Svendsen (1996) derived an expression for the breakage rate of a particle of volume $v$ into daughter particles of size $v f_{V}$ and $v\left(1-f_{V}\right)$ using energy arguments similar to those employed by Tsouris and Tavlarides. In this model, $f_{V}$ denotes the volume fraction $D^{3} / D_{0}^{3}$, where $D$ is the diameter of the daughter particle and $D_{0}$ is the diameter of the mother particle. As in the Tsouris and Tavlarides model, these authors assume binary break-up. The significant difference between the Luo and Svendsen model and its predecessors is that it gives both a "partial" breakage rate, that is, the breakage rate for a particle of size $v$ splitting into a particle of size $v f_{V}$ and its complement, and an overall breakage rate (the overall breakage rate is obtained by integrating the partial breakage rate from zero to one and multiplying by $1 / 2$ ). The previous surface energy models provided only an overall breakage rate. The expression for the daughter particle size distribution function is found by normalizing the partial breakage rate by the overall breakage rate.

The Luo and Svendsen daughter particle size distribution is determined from the expression

$$
f\left(v f_{V}, v\right)=\frac{2 \int_{\xi_{\min }}^{1}(1+\xi)^{2} \xi^{-11 / 3} \mathrm{e}^{-\chi_{c}} \mathrm{~d} \xi}{v \int_{0}^{1} \int_{\xi_{\min }}^{1}(1+\xi)^{2} \xi^{-11 / 3} \mathrm{e}^{-\chi_{c}} \mathrm{~d} \xi \mathrm{d} f_{V}},
$$

where $v=\pi D_{0}^{3} / 6$ is the volume of the mother particle, $v f_{V}=\pi D_{1}^{3} / 6$ is the volume of the resultant daughter particle, $\xi=D_{\mathrm{e}} / D$ is the size ratio between an eddy in the inertial subrange and the particle, and $\chi_{\mathrm{c}}$ is the critical dimensionless energy required for break-up

$$
\chi_{\mathrm{c}}=\frac{\bar{e}_{i}(d)}{\bar{e}(\lambda)}=\frac{12 C_{f} \sigma}{\beta \rho \epsilon^{2 / 3} D^{5 / 3} \xi^{11 / 3}} .
$$

As written, this model has one free parameter: the lower limit of integration in Eq. (67) (see Section 2 for a discussion of the sensitivity of this model to the upper limit of integration). This parameter defines the ratio between the particle diameter and the smallest eddies in the inertial subrange that can cause break-up. The minimum eddy size in the inertial subrange is taken to be between 11.4 and 31.4 times the Kolmogorov length scale (Tennekes and Lumley, 1972). 
The behavior of the Luo and Svendsen model is shown in Figs. 14-16. In Fig. 14, the dissipation rate of turbulent kinetic energy, $\epsilon$, is held fixed $\left(\epsilon=10 \mathrm{~m}^{2} \mathrm{~s}^{-3}\right)$ and the evolution of the model with increasing mother particle diameter can be seen. As the mother particle diameter is increased, the probability of equal breakage increases and the pdfs begin to flatten out. In all cases, however, the probability of breaking a mother particle into a very small particle and a complementary large particle far exceeds the probability of equal breakage. Fig. 15 shows the evolution of the model with dissipation rate for a fixed mother particle diameter. The pdfs flatten out and the probability of equal breakage increases significantly with increasing dissipation rate. Notice that the two curves representing $\epsilon=100 \mathrm{~m}^{2} \mathrm{~s}^{-3}$ and $\epsilon=1000 \mathrm{~m}^{2} \mathrm{~s}^{-3}$ are nearly identical, but the curve for the higher dissipation rate lies slightly below that for the lower dissipation rate.

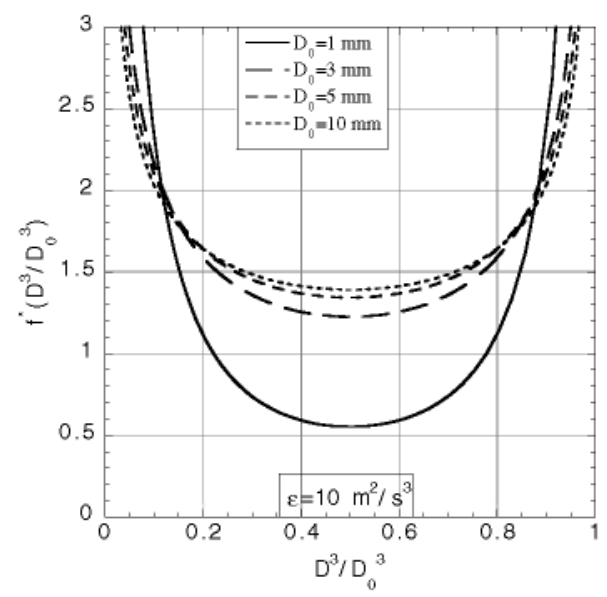

Fig. 14. Evolution of the Luo and Svendsen (1996) model with mother particle diameter. $\epsilon=10 \mathrm{~m}^{2} / \mathrm{s}^{3}$, $\sigma=0.072 \mathrm{~N} / \mathrm{m}, \rho=1000 \mathrm{~kg} / \mathrm{m}^{3}, \xi_{\min }=21.4 \eta / D_{0}$.

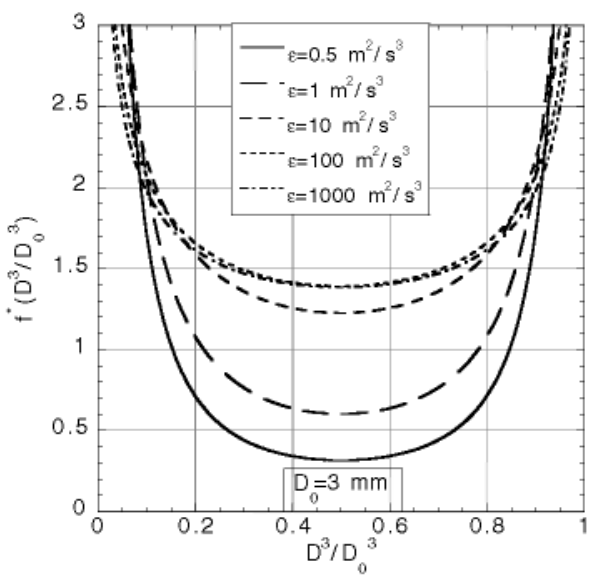

Fig. 15. Evolution of the Luo and Svendsen (1996) model with the dissipation rate of turbulent kinetic energy, $\epsilon$. $D_{0}=3 \mathrm{~mm}, \sigma=0.072 \mathrm{~N} / \mathrm{m}, \rho=1000 \mathrm{~kg} / \mathrm{m}^{3}, \xi_{\min }=21.4 \eta / D_{0}$. 


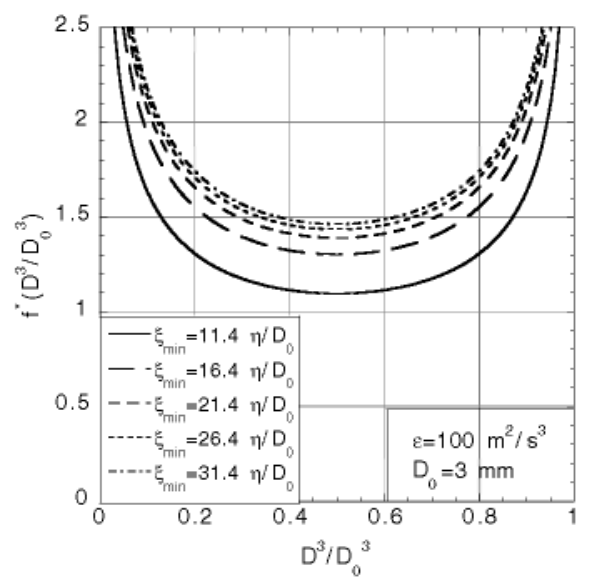

Fig. 16. Dependence of the Luo and Svendsen (1996) model on the parameter $\xi_{\min } . \epsilon=100 \mathrm{~m}^{2} \mathrm{~s}^{-3}, D_{0}=3 \mathrm{~mm}$, $\sigma=0.072 \mathrm{~N} / \mathrm{m}, \rho=1000 \mathrm{~kg} / \mathrm{m}^{3}$.

This non-monotonic evolution with $\epsilon$ does not match the physical situation. Fig. 16 depicts the sensitivity of this model to $\xi_{\min }$. The model is quite sensitive to the value of this parameter. For the conditions shown $\left(\epsilon=100 \mathrm{~m}^{2} \mathrm{~s}^{-3}, D_{0}=3 \mathrm{~mm}\right)$, the probability of equal breakage increases by $33 \%$ when $\xi_{\min }$ is changed from $11.4 \eta / D_{0}$ to $31.4 \eta / D_{0}$. The proper selection of $\xi_{\min }$ requires comparison with experimental data.

\subsubsection{Surface energy models based on stress balance}

Martínez-Bazán et al. (1999b) developed a model for the daughter bubble size distribution resulting from the break-up of air bubbles at the centerline of a high Reynolds number, turbulent water jet. This model assumes that when a mother particle breaks, two daughter particles are formed $\left(m\left(D_{0}\right)=2\right)$ with diameters $D_{1}$ and $D_{2}$. The validity of this assumption is supported by high-speed video images, given in Martínez-Bazán et al. (1999b). Diameters $D_{1}$ and $D_{2}$ are related through the conservation of mass. Therefore,

$$
D_{2}=D_{0}\left[1-\left(\frac{D_{1}}{D_{0}}\right)^{3}\right]^{1 / 3} \text {. }
$$

If the particle-splitting process were purely random, then a uniform distribution, like that chosen by Prince and Blanch (1990), and others, would be relevant to the physics of the problem. However, pressure fluctuations in homogeneous and isotropic turbulence are not uniformly distributed over all scales. This means that $\tau_{\mathrm{t}}$, given in Eq. (41), is not uniformly distributed and therefore the splitting process cannot be purely random. In fact, there is a distance, $D_{\min }$ over which $\tau_{\mathrm{t}}\left(D_{\min }\right)=\tau_{\mathrm{s}}\left(D_{0}\right)$. At this distance, the turbulent pressure fluctuations are exactly equal to the confinement forces for a mother particle of size $D_{0}$. The probability of breaking off a daughter particle with $D_{1}<D_{\min }=\left(12 \sigma /\left(\beta \rho D_{0}\right)\right)^{3 / 2} \epsilon^{-1}$ should therefore be zero.

The fundamental postulate of the Martínez-Bazán model is that the probability of splitting off a daughter particle of any size such that $D_{\min }<D_{1}<D_{0}$ is proportional to the difference between 
the turbulent stresses over a length $D_{1}$ and the confinement forces holding the mother particle of size $D_{0}$ together. For the formation of a daughter particle of size $D_{1}$, the difference in stresses is given by $\Delta \tau_{t 1}=\frac{1}{2} \rho \beta\left(\epsilon D_{1}\right)^{2 / 3}-6 \sigma / D_{0}$. For each daughter particle of size $D_{1}$, a complementary daughter particle of size $D_{2}$ is formed with a difference of stresses given by $\Delta \tau_{\mathrm{t} 2}=\frac{1}{2} \rho \beta\left(\epsilon D_{2}\right)^{2 / 3}-$ $6 \sigma / D_{0}$. The model states that the probability of forming a pair of complementary daughter particles of sizes $D_{1}$ and $D_{2}$ from splitting a mother particle of size $D_{0}$ is related to the product of the excess stresses associated with the length scales corresponding to each daughter particle size. That is,

$$
P\left(D_{1} / D_{0}\right) \propto\left[\frac{1}{2} \rho \beta\left(\epsilon D_{1}\right)^{2 / 3}-\frac{6 \sigma}{D_{0}}\right]\left[\frac{1}{2} \rho \beta\left(\epsilon D_{2}\right)^{2 / 3}-\frac{6 \sigma}{D_{0}}\right] .
$$

Relating $D_{1}$ and $D_{2}$ through the mass balance given by $\mathrm{Eq}$. (69) above yields

$$
P\left(D^{*}\right) \propto\left(\frac{1}{2} \rho \beta\left(\epsilon D_{0}\right)^{2 / 3}\right)^{2}\left[D^{* 2 / 3}-A^{5 / 3}\right]\left[\left(1-D^{* 3}\right)^{2 / 9}-A^{5 / 3}\right],
$$

where $D^{*}=D_{1} / D_{0}, \quad A=D_{\mathrm{c}} / D_{0}=\left(D_{\min } / D_{0}\right)^{2 / 5} . D_{\mathrm{c}}$ is the critical diameter defined as $D_{\mathrm{c}}=$ $(12 \sigma /(\beta \rho))^{3 / 5} \epsilon^{-2 / 5}$. Note the difference between the critical diameter, $D_{\mathrm{c}}$, and the minimum diameter, $D_{\text {min }}$. The critical diameter applies to the mother particle and defines the minimum particle size for a given dissipation rate of turbulent kinetic energy for which break-up can occur. The minimum diameter, on the other hand, applies to the daughter particles and defines the distance over which the turbulent normal stresses just balance the confinement forces of a mother particles of size $D_{0}$. The minimum diameter, therefore, gives the minimum length over which the underlying turbulence can pinch off a piece of the mother particle. This length is not arbitrarily selected; rather, its determination is based on kinematics.

This model assumes that the size of the particles is in the inertial subrange. Therefore, it implies that $D_{\min } \leqslant D_{1} \leqslant D_{\max }$ provided that $D_{\min }>\eta$, where $\eta$ is the Kolmogorov length scale of the underlying turbulence. Otherwise, $D_{\min }$ is taken to be equal to $\eta$. Also, note that $D_{\max }$ and $D_{\min }$ are related through $\mathrm{Eq}$. (69). No assumption needs to be made about the minimum and maximum eddy size that can cause particle break-up. All eddies with sizes between the Kolmogorov scale and the integral scale are taken into account.

The daughter particle probability density function can be obtained from the expression given in Eq. (71) by utilizing the normalization condition $\int_{D_{\text {max }}^{m}}^{D_{\text {max }}^{*}} P\left(D^{*}\right) \mathrm{d}\left(D^{*}\right)=1$. The pdf of the ratio of diameters $D^{*}=D_{1} / D_{0}, f^{*}\left(D^{*}\right)$, can then be written as

$$
f^{*}\left(D^{*}\right)=\frac{\left[D^{* 2 / 3}-A^{5 / 3}\right]\left[\left(1-D^{* 3}\right)^{2 / 9}-A^{5 / 3}\right]}{\int_{D_{\min }^{\max }}^{D_{\max }}\left[D^{* 2 / 3}-A^{5 / 3}\right]\left[\left(1-D^{* 3}\right)^{2 / 9}-A^{5 / 3}\right] \mathrm{d}\left(D^{*}\right)} .
$$

Note that $f\left(D_{1}, D_{0}\right)=f^{*}\left(D^{*}\right) / D_{0}$.

The Martínez-Bazán model, Eq. (72), is plotted in Figs. 17 and 18. In Fig. 17, the size of the mother particle is $D_{0}=3 \mathrm{~mm}$ and the dissipation rate of turbulent kinetic energy, $\epsilon$, is varied over three orders of magnitude. In Fig. 18, $\epsilon=1000 \mathrm{~m}^{2} \mathrm{~s}^{-3}$ and the diameter of the mother particle is varied from 0.4 to $3 \mathrm{~mm}$. In all cases, the peak of the pdf is located at $D^{*}=0.8$. This value corresponds to the formation of two daughter particles of the same volume. This result differs from the collision-based phenomenological models for the daughter particle size distribution, 


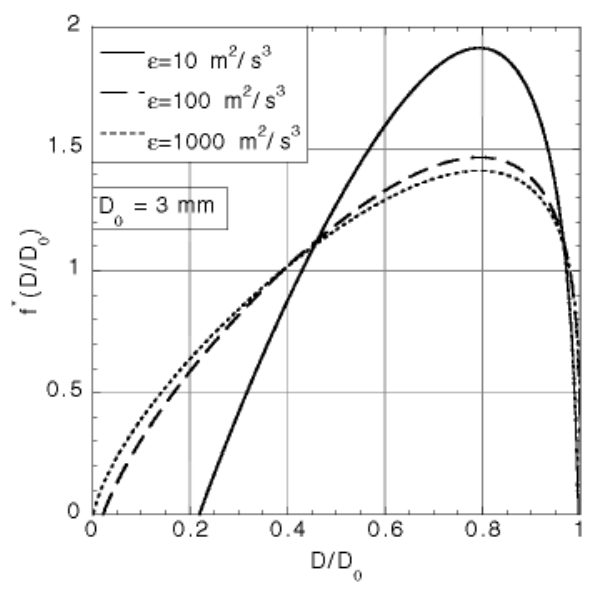

Fig. 17. Dimensionless daughter particle probability density function for the Martínez-Bazán model, indicating the evolution of the pdf with the dissipation rate of turbulent kinetic energy, $\epsilon\left(\mathrm{m}^{2} \mathrm{~s}^{-3}\right) . D^{*}=D_{1} / D_{0}$. The mother particle diameter, $D_{0}$ for each curve is $3 \mathrm{~mm} . \sigma=0.072 \mathrm{~N} / \mathrm{m}, \rho=1000 \mathrm{~kg} / \mathrm{m}^{3}$.

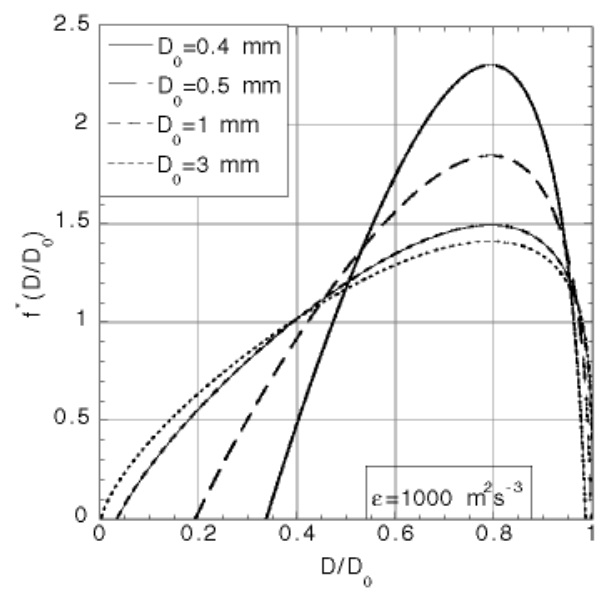

Fig. 18. Dimensionless daughter particle probability density function for the Martínez-Bazán model, indicating the evolution of the pdf with the mother particle diameter, $D_{0} . D^{*}=D_{1} / D_{0}$. $\epsilon$ for each curve is $1000 \mathrm{~m}^{2} \mathrm{~s}^{-3}$. $\sigma=0.072 \mathrm{~N} / \mathrm{m}, \rho=1000 \mathrm{~kg} / \mathrm{m}^{3}$.

although it agrees with the hybrid model proposed by Konno. Notice that the size pdf becomes wider as either $\epsilon$ or $D_{0}$ are increased. This behavior is intuitive. A greater value of $\epsilon$ means that the underlying turbulence is stronger, leading to an increase in the fraction of the overall energy contained in smaller scales. In other words, as $\epsilon$ increases, $D_{\min }$ decreases, making the formation of smaller daughter particles more probable. Similarly, as $D_{0}$ increases, a larger fraction of the eddies in the inertial subrange have sizes which are smaller than the mother particle. Therefore, the probability of creating smaller daughter particles increases.

To summarize, statistical models for the daughter particle size distribution lack physical support. The models based eddy collision arguments rely on the assumption that turbulence consists 
of a collection of eddies that can be treated like molecules in the kinetic theory of gases. Further, they require the specification of the minimum and maximum eddy sizes that are capable of causing particle break-up (beyond the requirement that these eddies are within the inertial subrange). The models can be quite sensitive to these parameters. In the following section, we compare the most popular of these phenomenological models for $f\left(D, D_{0}\right)$, along with Konno's hybrid model, with experimental data obtained from the break-up of air bubbles immersed in a high Reynolds number, submerged water jet (for further comparisons, see Martínez-Bazán et al. (1999a,b)).

\section{Comparison with experimental data}

We conducted an experimental investigation of the fragmentation of an air cavity injected into a high Reynolds number, submerged, water jet. A detailed description of the experimental facility and of the measurement techniques employed is given in Martínez-Bazán (1998) and in MartínezBazán et al. (1999a,b). Only the key features of the experiment will be presented here.

First, the mean velocities and turbulence properties of a submerged, high Reynolds number water jet were measured using a combination of hot-wire and laser Doppler anemometry. Subsequently, air was injected continuously through a small-diameter, hypodermic needle located at the centerline in the fully developed region of the water jet. A variety of needle diameters were used in order to vary the bubble size distribution independently of the flow conditions. Similarly, various jet Reynolds numbers were tested to systematically vary the level of background turbulence for each of the needle diameters employed. The injection velocity of the air was selected to match the mean centerline velocity of the water jet at the point of injection. Since the air bubbles were convected at the local mean velocity of the water jet, the break-up was caused solely by to the turbulent fluctuations existing at the surface of each bubble. Digital images of the break-up process were captured at successive, downstream locations from the air injection point, until a location was reached at which the underlying turbulence was no longer strong enough to continue to break the bubbles. Using the images collected, the bubble size distribution was determined at each measurement location. As the air bubbles were fragmented and convected downstream, their radial dispersion was limited to a region about the centerline of the water jet that was less than $30 \%$ of the local jet diameter. Therefore, the turbulence encountered by the air bubbles was nearly spatially uniform and isotropic.

The results of these experiments were compared to the models for turbulent particle break-up developed by Konno et al. (1980, 1983), Tsouris and Tavlarides (1994), Luo and Svendsen (1996), and Martínez-Bazán et al. (1999a,b). The expressions for $g(D), m\left(D_{0}\right)$, and $f\left(D, D_{0}\right)$ derived by these authors were substituted into the following equation:

$$
\frac{\partial(U n)}{\partial x}=\int_{D}^{\infty} m\left(D_{0}\right) f\left(D, D_{0}\right) g\left(D_{0}\right) n\left(D_{0}, t\right) \mathrm{d} D_{0}-g(D) n(D, t),
$$

where $U(x)$ is the mean centerline velocity of the water jet, and the other terms are as defined in Section 1. Note that $U(x)$ is the mean convective velocity of all bubbles, $\mathbf{v}(D, \mathbf{x}, t)$, regardless of their size. Eq. (73) was integrated to determine the downstream evolution of the cumulative bubble volume pdf for each of these models. 
A comparison of the model predictions with our experimental data is shown in Figs. 19-22. The figures portray the evolution of the cumulative bubble volume pdf with downstream distance. For this set of data, air was injected at 15 nozzle diameters downstream from the water nozzle exit. The hypodermic needle used to inject the air had an inner diameter of $0.394 \mathrm{~mm}$ and the air injection velocity was $9.84 \mathrm{~m} / \mathrm{s}$. The jet Reynolds number based on the diameter of the water nozzle and on the exit velocity of the water was 25,500 . The dissipation rate of turbulent kinetic energy at the air injection point, $\epsilon_{0}$, was $1000 \mathrm{~m}^{2} \mathrm{~s}^{-3}$. The initial condition used in Eq. (73) was the bubble size distribution

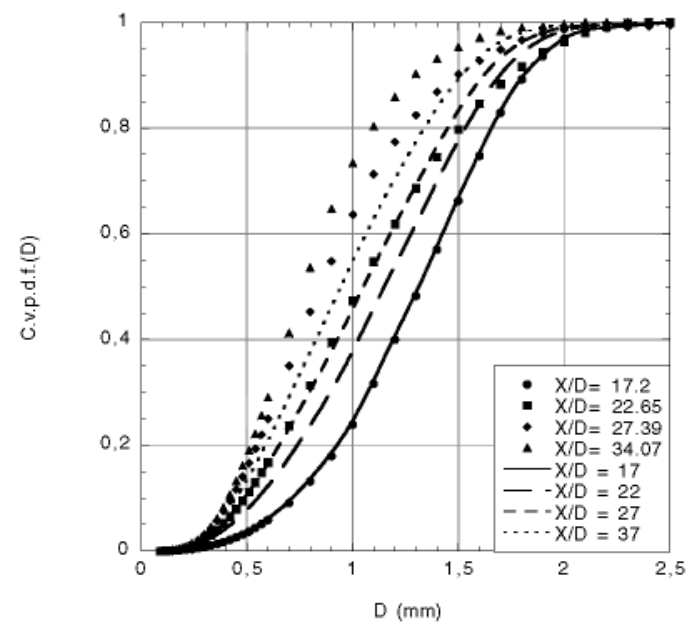

Fig. 19. Downstream evolution of the cumulative volume probability density function $(\operatorname{Cvpdf}(D))$ for air bubbles injected at the centerline of a turbulent water jet. The symbols represent experimental data. The lines represent the predictions of the Tsouris and Tavlarides (1994) model.

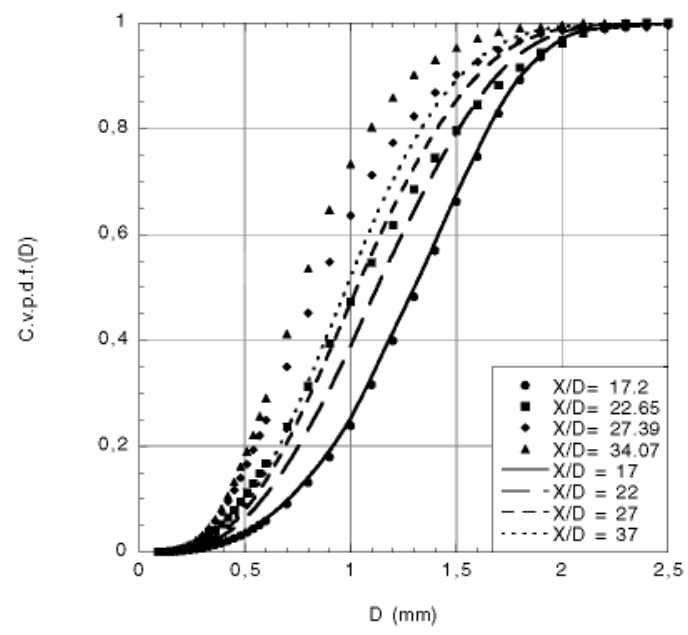

Fig. 20. Downstream evolution of the cumulative volume probability density function $(\operatorname{Cvpd}(D))$ for air bubbles injected at the centerline of a turbulent water jet. The symbols represent experimental data. The lines represent the predictions of the Luo and Svendsen (1996) model. 


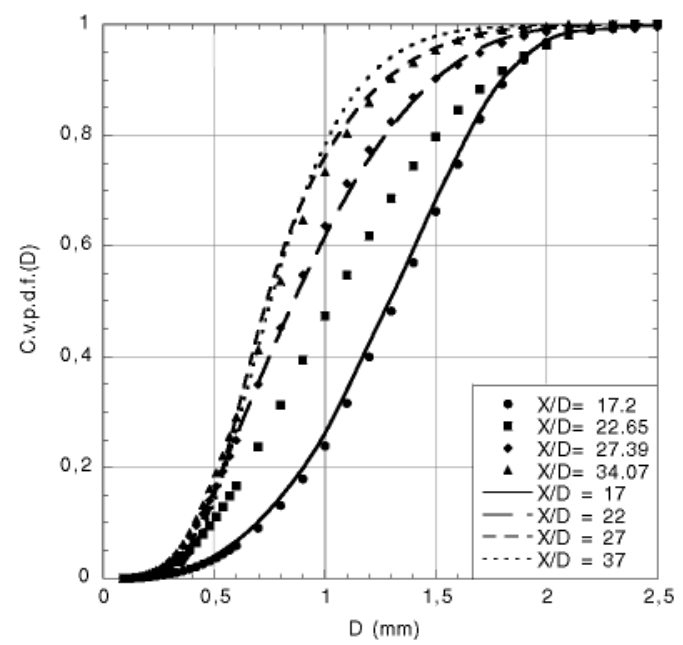

Fig. 21. Downstream evolution of the cumulative volume probability density function $(\operatorname{Cvpd} f(D))$ for air bubbles injected at the centerline of a turbulent water jet. The symbols represent experimental data. The lines represent the predictions of the Konno et al. $(1980,1983)$ model.

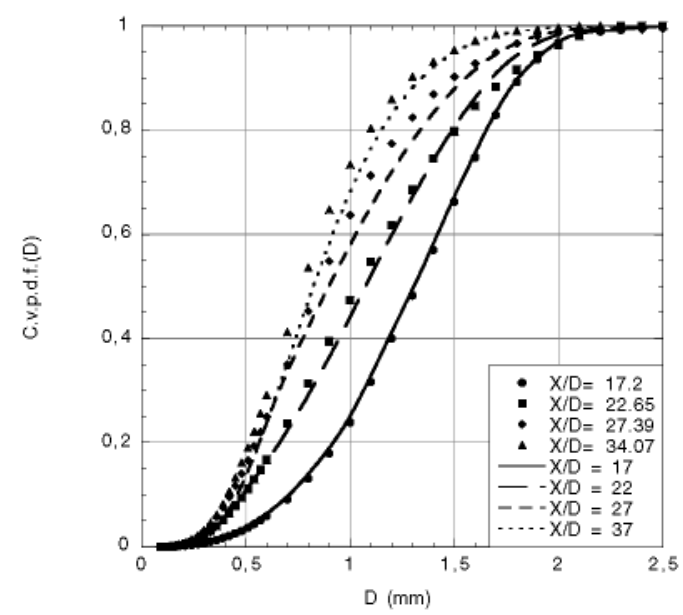

Fig. 22. Downstream evolution of the cumulative volume probability density function $(C v p d f(D))$ for air bubbles injected at the centerline of a turbulent water jet. The symbols represent experimental data. The lines represent the predictions of the Martínez-Bazán et al. (1999a,b) model.

existing 2.2 water nozzle diameters downstream from the air injection point $\left(X / D_{j}=17.2\right)$. In the figures, experimental results are displayed as symbols, model predictions are displayed as lines.

It is immediately apparent that the collision-based models of Tsouris and Tavlarides and Luo and Svendsen, shown in Figs. 19 and 20 fail to capture the downstream evolution of the cumulative volume pdf. The relationships for $f\left(D, D_{0}\right)$ in each of these models favor the formation of a very small daughter bubble and its complementary very large daughter bubble for each break-up event. Because of this assumption, both of these models underpredict the fraction of 
intermediately sized bubbles seen in the cumulative volume pdf. Although the Luo and Svendsen relation for $f\left(D, D_{0}\right)$ does depend on $\epsilon$, it does not exhibit better agreement with this experimental data set than the Tsouris and Tavlarides model, which has no dependence on $\epsilon$. Neither of these models adequately capture the physics of this process.

The results obtained from the model of Konno et al. are shown in Fig. 21. As described in the previous sections, Konno utilized a collision-based model for $g(D)$ similar to Coulaloglou and Tavlarides; however, his hybrid model for the daughter particle pdf differs from the collisionbased models for $f\left(D, D_{0}\right)$ in that it predicts a maximum probability for the formation of two, equally sized, daughter bubbles. This model is well-approximated by the universal function given in Eq. (57), which has no dependence on $\epsilon$. As a result, Konno's model over-predicts break-up as the bubbles are convected into regions of successively lower dissipation rates. This is seen in the figure by the fact that the lines representing Konno's model lie above the points representing the experimental data for intermediate bubble diameters.

The predictions of the Martínez-Bazán et al. model are shown in Fig. 22. Both the expression for $g(D)$ and the expression for $f\left(D, D_{0}\right)$ in this model are based on the kinematics existing at the surface of each bubble. Although this model is conceptually and mathematically simpler than the others, it predicts the experimental results much more accurately. The model seems to slightly under-predict the formation of intermediately sized bubbles at $X / D_{j}=27.39$ and slightly underpredict the formation of small bubbles at $X / D_{j}=34.04$; however, the overall agreement with the experimental data is excellent. Further comparisons of this model with experimental data are shown in Martínez-Bazán et al. (1999b).

\section{Conclusions}

We have comparatively described various models proposed in the literature for the break-up of a fluid lump immersed into a fully developed turbulent flow. Comparison between the predictions resulting from these models and experimental data have shown that the simple phenomenological models proposed by Martínez-Bazán et al. (1999a,b), based purely on kinematic considerations, better predict the experimental data while simultaneously requiring fewer closure parameters.

\section{Acknowledgements}

The authors gratefully acknowledge the support of the ONR under contract \# N00014-96-10213 and \# N00014-7-1-0305, and the Spanish Ministry of Education, project number PB98-0142C04-02. We would also like to thank Javier Rodríguez-Rodríguez for his assistance comparing these models to our experimental data.

\section{References}

Batchelor, G.K., 1956. The Theory of Homogeneous Turbulence. Cambridge University Press, London.

Chatzi, E., Garrielides, A.D., Kiparissides, C., 1989. Generalized model for prediction of the steady-state drop size distributions in batch stirred vessels. Ind. Eng. Chem. Res. 28, 1704-1711. 
Chatzi, E., Kiparissides, C., 1992. Dynamic simulation of bimodal drop size distributions in low-coalescence batch dispersion systems. Chem. Eng. Sci. 47, 445-456.

Collins, S.B., Knudsen, J.G., 1970. Drop-size distributions produced by turbulent pipe flow of immiscible liquids. AIChE J. 16, 1072-1080.

Coulaloglou, C.A., Tavlarides, L.L., 1977. Description of interaction processes in agitated liquid-liquid dispersions. Chem. Eng. Sci. 32, 1289-1297.

Eastwood, C., Cartellier, A., Lasheras, J.C., 2000. The break-up time of a droplet in a fully developed turbulent flow. In: Advances in Turbulence VIII, Proceedings of the Eight European Turbulence Conference. Barcelona, pp. 573576.

Hesketh, R.P., Etchells, A.W., Russell, T.W.F., 1991. Bubble breakage in pipeline flow. Chem. Eng. Sci. 46, 1-9.

Hinze, J.O., 1955. Fundamentals of the hydrodynamics mechanisms of splitting in dispersion process. AIChE J. 1, 289295.

Hsia, A.M., Tavlarides, L.L., 1983. Simulation analysis of drop breakage, coalescence, and micromixing in liquidliquid stirred tanks. Chem. Eng. J. 26, 189-199.

Kolev, N.I., 1993. Fragmentation and coalescence dynamics in multiphase flows. Exp. Thermal Fluid Sci. 6, $211-251$.

Kolmogorov, A.N., 1949. On the breakage of drops in a turbulent flow. Dokl. Akad. Navk. SSSR 66, 825-828.

Konno, M., Matsunaga, Y., Arai, K., Saito, S., 1980. Simulations model for break-up process in an agitated tank. J. Chem. Eng. Jpn. 13, 67-73.

Konno, M., Aoki, M., Saito, S., 1983. Scale effect on break-up process in liquid-liquid agitated tanks. J. Chem. Eng. Jpn. 16, 312-319.

Lasheras, J.C., Hopfinger, E.J., 2000. Liquid jet instability and atomization in a coaxial gas stream. Ann. Rev. Fluid Mech. 32, 275-308.

Lee, C.H., Erickson, L.E., Glasgow, L.A., 1987. Dynamics of bubble size distribution in turbulent gas-liquid dispersions. Chem. Eng. Commun. 61, 181-195.

Longuet-Higgins, M.S., 1992. The crushing of air cavities in a liquid. Proc. R. Soc. London, Ser. A 439, 611-626.

Luo, H., Svendsen, F., 1996. Theoretical model for drop and bubble break-up in turbulent dispersions. AIChE J. 42, $1225-1233$.

Martínez-Bazán, C., 1998. Splitting and dispersion of bubbles by turbulence. Ph.D. thesis, University of California, San Diego.

Martínez-Bazán, C., Montañes, J.L., Lasheras, J.C., 1999a. On the break-up of an air bubble injected into a fully developed turbulent flow. Part I: Break-up frequency. J. Fluid Mech. 401, 157-182.

Martínez-Bazán, C., Montañes, J.L., Lasheras, J.C., 1999b. On the break-up of an air bubble injected into a fully developed turbulent flow. Part II: Size pdf of the resulting daughter bubbles. J. Fluid Mech. 401, 183-207.

Nambiar, D.K.R., Kumar, R., Das, T.R., Gandhi, K.S., 1992. A new model for the breakage frequency of drops in turbulent stirred dispersions. Chem. Eng. Sci. 47, 2989-3002.

Narsimhan, G., Gupta, J.P., Ramkrishna, D., 1979. A model for transitional breakage probability of droplets in agitated lean liquid-liquid dispersions. Chem. Eng. Sci. 34, 257-265.

Novikov, E.A., Dommermuth, D.G., 1997. Distribution of droplets in a turbulent spray. Phys. Rev. E 56, 54795482.

Prince, M.J., Blanch, H.W., 1990. Bubble coalescence and break-up in air-sparged bubble columns. AIChE J. 36, 14851499.

Randolph, A.D., 1969. Effect of crystal breakage on crystal size distribution in a mixed suspension crystallizer. Ind. Eng. Chem. Fundamentals 59, 58 .

Ross, S.L., Ph.D. dissertation. University of Michigan, Ann Arbor, MI, 1971.

Ross, S.L., Curl, R.L., 1973. Measurement and models of the dispersed phase mixing process. In: Joint Chemical Engineering Conference, Paper 29b, Vancouver.

Ross, S.L., Verhoff, F.H., Curl, R.L., 1978. Droplet breakage and coalescence processes in an agitated dispersion. 2. Measurement and interpretation of mixing experiments. Ind. Eng. Chem. Fundamentals 17, 101-108.

Tennekes, H., Lumley, J.L., 1972. A First Course in Turbulence. MIT Press, Cambridge, MA.

Tsouris, C., Tavlarides, L.L., 1994. Breakage and coalescence models for drops in turbulent dispersions. AIChE J. 40 , 395-406. 
Valentas, K.J., Bilous, O., Amundson, N.R., 1966. Analysis of breakage in dispersed phase systems. Ind. Eng. Chem. Fundamentals 5, 271-279.

Verhoff, F.H., Ross, S.L., Curl, R.L., 1977. Breakage and coalescence processes in an agitated dispersion. Experimental system and data reduction. Ind. Eng. Chem. Fundamentals 16, 371-377.

Williams, F.A., 1985. Combustion Theory, second ed. Benjamin/Cummings, Menlo Park, CA. 